\title{
Creación y estudio de las propiedades psicométricas del cuestionario de socialización parental TXP
}

\section{Creation of the TXP parenting questionnaire and study of its psychometric properties}

\author{
Ana Benito*,**, Gema Calvo*, Matías Real-López*******, María José Gallego***,****, \\ Sonia Francés*, Ángel Turbi*****, Gonzalo Haro*****. \\ *Grupo Investigación TXP, Departamento de Medicina, Universidad Cardenal Herrera-CEU, CEU Universities, Castellón, \\ España; **Unidad Salud Mental Torrent, Hospital General, Valencia, España; ***Servicio de Psiquiatría, Consorcio \\ Hospitalario Provincial, Castellón; ****Facultad de Ciencias de la Salud, Universidad Jaume I, Castellón; ***** Facultad de \\ Psicología, Universidad Católica San Vicente Mártir, Valencia.
}

\section{Resumen}

El estilo parental de socialización se relaciona con trastornos de conducta (TC) y trastornos relacionados con sustancias (TRS) en adolescentes, con diferencias según el contexto cultural. Se diseñó mediante método Delphi un cuestionario con dos versiones (Cuestionario de socialización parental TXP-A para adolescentes y $T X P$-C para cuidador principal) para evaluar en población española las prácticas de socialización parental relacionadas con TC y TRS. Se validó en una muestra comunitaria de 631 adolescentes entre 14 y 16 años y sus cuidadores. Los resultados recomiendan un cuestionario TXP-A de 29 ítems y estructura bifactorial: afecto-comunicación y controlestructura, mostrando alta fiabilidad interna (alfa de Cronbach $=0,89$ ) y test-retest (coeficiente de correlación intraclase $=0,94$ ). Ambos factores correlacionan con TRS $(\mathrm{r}=0,273 ; \mathrm{p}<0,001)$ y con la mayoría de las dimensiones psicopatológicas estudiadas. La puntuación total y afectocomunicación se relacionan con el trastorno disocial $(\mathrm{t}=3,259 ; \mathrm{p}=0,001)$ y su gravedad $(\mathrm{r}=-0,119 ; \mathrm{p}=0,003)$. La fiabilidad interjueces entre adolescentes y cuidadores es baja, en parte porque el TXP-C, de 16 ítems, presenta una estructura bifactorial diferente: afecto-comunicación y valores prosociales. La fiabilidad interna (alfa de Cronbach $=0,87$ ) y test-retest (coeficiente de correlación intraclase $=0,94$ ) del $T X P-C$ son altas. La puntuación total y afecto-comunicación se relacionan con el trastorno disocial $(\mathrm{t}=2,586 ; \mathrm{p}=0,010)$ pero no discrimina según el TRS. En conclusión, el cuestionario TXP-A para adolescentes parece un instrumento fiable, válido y sin sesgos que evalúa la percepción de las prácticas de socialización parental, relacionando mayores puntuaciones en afecto-comunicación y control-estructura con menor psicopatología y consumo de alcohol y drogas. El TXP-C también parece fiable y sin sesgos, pero muestra menos evidencias de validez respecto al consumo de sustancias y la psicopatología.

Palabras clave: Socialización parental; Trastornos de conducta; Trastornos relacionados con sustancias; Afecto-comunicación; Controlestructura; Valores prosociales.

\begin{abstract}
Parenting is linked to conduct disorders (CD) and substance related disorders (SRD) in adolescents, but with differences according to cultural context. A questionnaire with two versions (parenting questionnaire TXP-A for adolescents and TXP-C for primary caregivers) was designed using the Delphi method to evaluate parenting practices related to $\mathrm{CD}$ and $\mathrm{SRD}$ in a Spanish population. It was validated in a community sample of 631 adolescents aged between 14 and 16 and their caregivers. Results suggest a 29-item TXP-A questionnaire with bifactorial structure: affection-communication and control-structure, with high internal (Cronbach's alpha $=0.89$ ) and test-retest (intraclass correlation coefficient $=0.94$ ) reliabilities. Both factors are related to SRD $(r=0.273, p<0.001)$ and with most of the psychopathological dimensions studied. The total score and affection-communication are related to dissocial disorder $(\mathrm{t}=3.259, \mathrm{p}=0.001)$ and its severity $(\mathrm{r}=-$ $0,119 ; \mathrm{p}=0.003)$. Inter-observer reliability between adolescents and caregivers is low, in part because the 16-item TXP-C has a different bifactorial structure: affection-communication and prosocial values. $T X P-C$ s internal (Cronbach's alpha $=0.87$ ) and test-retest (intraclass correlation coefficient $=0.94$ ) reliabilities are high. The total score and affection-communication were related to dissocial disorder $(\mathrm{t}=2.586$; $\mathrm{p}=0.010)$ but $T X P$ - $C$ did not discriminate according to SRD.

In conclusion, the TXP-A questionnaire for adolescents seems to be a reliable, valid and unbiased instrument that evaluates the perception of parenting practices, relating higher affection-communication and control-structure to less psychopathology and alcohol and drug use. $T X P$-C also seems to be reliable and unbiased, but shows less evidence of validity regarding substance use and psychopathology. Keywords: Parenting; Conduct disorders; Substance related disorders; Affection-communication; Control-structure; Prosocial values.
\end{abstract}

Recibido: Junio 2017; Aceptado: Octubre 2017.

Enviar correspondencia a:

Prof. Gonzalo Haro. Programa de Patología Dual Grave. Hospital Provincial de Castellón. Avda. Dr. Clará, 19.

12002. Castellón de la Plana (Castellón). España. Teléfono: 964359700. E-mail: gonzalo.haro@uchceu.es 


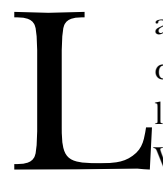

a familia es un contexto principal para el aprendizaje de valores, normas y costumbres durante la infancia y la adolescencia (Visser, de Winter, Vollebergh, Verhulst y Reijneveld, 2013) y uno de los agentes que puede influir en el aumento, mantenimiento o disminución de los síntomas psicopatológicos (Rosa-Alcázar, Parada-Navas y Rosa-Alcázar, 2014). La mayoría de los estudios sobre la familia se han centrado en los progenitores y, más en concreto, en los estilos educativos o socialización parental que emplean éstos (Rosa-Alcázar et al., 2014).

El modelo tradicional de socialización familiar de Baumrind (1991) consta de dos dimensiones: afecto-comunicación (comportamiento de los padres hacia los hijos mediante el cual los niños sienten que son amados y se sienten aceptados como individuos dentro de la familia) y exigencias-control (grado de intensidad o tipo de influencia de los padres en la conducta de los hijos), clasificando a los progenitores en los estilos democrático (alto control y alta aceptación y sensibilidad, enfatiza tanto el respeto a la individualidad del niño como la inculcación de valores sociales), autoritario (alta exigencia y control y baja sensibilidad, enfatiza el control y la obediencia) y permisivo/indulgente (baja exigencia y control y alta aceptación y sensibilidad, enfatiza la autoexpresión y la auto-regulación) (Bersabé, Fuentes y Motrico, 2001; Martínez, Díaz, Salazar y Duron, 2014). Maccoby y Martin (1983) añaden el estilo negligente (baja exigencia-control y bajo afecto-comunicación). A lo largo del tiempo se han propuesto otros modelos y dimensiones de socialización parental, como el Modelo Circumplejo Tridimensional de Olson (1988), con tres dimensiones: cohesión, flexibilidad y comunicación, clasificando a las familias en 25 tipos (Rees y Valenzuela, 2003).

A pesar de la diversidad de modelos, se considera que existen dos dimensiones básicas de los estilos educativos (Sansinenea y Sansinenea, 2004): una relacionada con el tono emocional de la relación y la comunicación (afecto y comunicación, aceptación/rechazo, calor/frialdad, afecto/hostilidad, proximidad/distanciamiento) y otra con las conductas puestas en juego para controlar y guiar el comportamiento de los hijos (control y disciplina). Estas prácticas suelen estar presentes en todas las familias, dependiendo el uso de una u otra de la situación específica en la que se aplica, aunque suele existir un estilo dominante que se pone en marcha con mayor frecuencia (Rodríguez y Torrente, 2003). Aunque la autonomía aumenta durante la transición a la adolescencia, los progenitores continúan siendo importantes para el desarrollo del adolescente (Visser et al., 2013), percibiendo los adolescentes que sus padres utilizan patrones relativamente estables de educación (Rodríguez y Torrente, 2003).

La investigación a lo largo de muchas décadas ha destacado la importancia del estilo parental para el desarrollo de conductas antisociales (Waller, Gardner y Hyde, 2013). $\mathrm{El}$ afecto negativo correlaciona con la conducta agresiva y los problemas de atención y del comportamiento, mientras que el estilo autoritario lo hace con depresión y ansiedad, conducta delictiva y otros problemas internalizantes (Rosa-Alcázar et al., 2014). La violencia y la negligencia son dos de los factores que mejor predicen los trastornos de conducta y la conducta antisocial (Holmes, Slaughter y Kashani, 2001). Monitorización, calidez y control conductual están asociados con niveles más bajos de problemas de conducta en la adolescencia (Trudeau, Mason, Randall, Spoth y Ralston, 2012). El afecto y apoyo junto con la disciplina moderada y consistente pueden inhibir los problemas de conducta (Loke y Mak, 2013). Además, el afecto y la conexión familiar actúan como factores protectores ante muchos de los comportamientos de riesgo de los adolescentes (Loke y Mak, 2013). En esta línea, la monitorización parental se asocia con efectos positivos en el uso de sustancias por adolescentes, con una reducción del consumo y una menor probabilidad de tener pares consumidores; protegiendo también a los adolescentes de la influencia potencialmente negativa de los pares (Tornay et al., 2013).

El estilo democrático es el que ha mostrado mayores beneficios para el desarrollo de los hijos (Fernández, 2009), siendo reconocido como el más beneficioso en la sociedad norteamericana (Lidner, 2013), donde los estilos democrático y autoritario actuaban como factor de protección para el consumo de sustancias mientras que los estilos indulgente y negligente suponían un factor de riesgo (Martínez, Fuentes, García y Madrid, 2013). Sin embargo, en grupos étnicos minoritarios, en contextos diferentes al anglosajón y en familias con un nivel socio-económico bajo, el estilo educativo autoritario basado en la imposición y no en el afecto parental parece ser un estilo más adecuado (Fuentes, García, García y Alarcón, 2015). En España, diferentes estudios asocian el estilo indulgente basado en el afecto con los mejores resultados en el ajuste psicosocial de los hijos (Fuentes et al., 2015). Un número destacado de publicaciones coincide en que los adolescentes de padres indulgentes obtienen iguales o mejores puntuaciones en diferentes criterios de ajuste que los hijos con familias democráticas (Pérez, 2012) y en que el estilo indulgente actúa como protección para el consumo de sustancias en adolescentes (Martínez et al., 2013). Esto contrasta con los estudios que encuentran en adolescentes españoles que una alta permisividad se relaciona con el consumo de alcohol (Mezquita et al., 2006).

Se han desarrollado numerosos instrumentos para evaluar los estilos parentales, siendo ejemplos significativos: Inventario para hijos/as del comportamiento parental (CRPBI; Schaefer, 1965); Escala de Clima Social en la Familia (Moos, Moos y Trichett, 1984), FACES III (Escala de Evaluación de la Adaptabilidad y Cohesión Familiar Tercera Versión; Olson, Portner y Lavee, 1985); Parental Authority Questionnaire (PAQ; Buri, 1991), Alabama Child Parenting Questionnaire (ACPQ; Shelton, Frick y Wootton, 1996), Escala de socialización pa- 
rental en la adolescencia (ESPA29; Musitu y García, 2001), Escala para la evaluación del estilo parental (Oliva, Parra, Sánchez-Quija y López, 2007) y Cuestionario de estilo educativo percibido (EMBU; Arrindell et al., 2005). Destacar como limitación de estos instrumentos que sólo el ESPA29 y la Escala para la evaluación del estilo parental han sido desarrollados en nuestro medio cultural. Además, estos dos instrumentos sólo evalúan la percepción del adolescente pero no la parental, lo que justificaría la creación de un instrumento que evalúe ambas perspectivas en nuestro medio.

Según Bersabé et al. (2001), los cuestionarios sobre los estilos educativos parentales presentan problemas de contenido (evalúan intenciones u opiniones de los padres en lugar de prácticas concretas o los ítems se formulan de forma genérica o en tercera persona, lo que favorece la deseabilidad social y los hace ambiguos) y metodológicos (muchos no especifican las edades de los hijos a las que van dirigidos; algunos no dan información de sus propiedades psicométricas, ni de la escala de respuesta utilizada; el número de ítems presenta una gran variabilidad, siendo en algunos casos excesivo; muchos sólo recogen la opinión de los progenitores pero no la percepción de los hijos del estilo educativo que reciben). Además, dada la escasez de cuestionarios de estilos educativos en España, en muchos casos los investigadores han tenido que adaptar o traducir los cuestionarios validados en otras poblaciones (Bersabé et al., 2001).

Por otro lado, en la práctica clínica con adolescentes es fundamental el trabajo con los padres. Los programas multicomponente han mostrado su eficacia (Romero, Rodríguez, Villar y Gómez-Fraguela, 2017). Para decidir qué componentes incluir en dichos programas es necesario conocer en qué factores de la socialización parental hay que focalizar la intervención para prevenir o moderar determinadas patologías. Así, es mucho más operativo incluir intervenciones sobre prácticas parentales concretas que sobre el estilo parental global. No obstante, los componentes de los estilos educativos se relacionan de diferente manera con los diferentes síntomas psicopatológicos. Por ejemplo, con los problemas de conducta se han relacionado la monitorización, la calidez y el control (Trudeau et al., 2012) y con el consumo de drogas los grados de cohesión, adaptabilidad, fortalezas y unión familiares y la felicidad marital de los padres (Rees y Valenzuela, 2003). Por ello, para diseñar intervenciones clínicas nos parece más operativo un cuestionario basado en prácticas parentales concretas consideradas por los expertos relevantes en la aparición y mantenimiento de estas patologías que los que evalúan el estilo parental global.

Además, existen diferencias en las relaciones de la socialización parental y las patologías según el contexto cultural donde se produce la socialización, siendo unas prácticas más efectivas que otras en determinados contextos. Por ejemplo, en grupos étnicos minoritarios, en contextos di- ferentes al anglosajón e incluso en familias con un nivel socio-económico bajo, la imposición podría funcionar mejor que el afecto, mientras que en España ocurriría al revés (Fuentes et al., 2015). Esto denota la importancia de usar instrumentos diseñados pensando en el contexto cultural en el que van a ser usados.

Todas estas razones, junto a las limitaciones encontradas en los cuestionarios existentes, justifican la creación de nuestro cuestionario. Para ello nos planteamos como objetivo de este estudio el diseño y estudio de las propiedades psicométricas en una muestra comunitaria de un cuestionario que evalúe en población española las prácticas de socialización parental que influyen en la aparición y mantenimiento de los trastornos de conducta (TC) y los trastornos relacionados con sustancias (TRS), considerando tanto la percepción del adolescente como la de su cuidador principal. La hipótesis 1 es que el cuestionario tendrá una estructura bifactorial (afecto-comunicación y control-disciplina), siendo un objetivo secundario comprobar cómo se relacionan los factores y las puntuación total con la personalidad, la psicopatología y el consumo de alcohol y drogas, así como evaluar si permiten diferenciar a la población general de los adolescentes con trastorno disocial o con trastorno negativista desafiante. La hipótesis 2 es que puntuaciones bajas en ambos factores y total se relacionarán con mayor psicopatología, mostrando el cuestionario evidencias de validez convergente, discriminante y de criterio.

\section{Métodos}

\section{Diseño del cuestionario}

El cuestionario fue diseñado en tres etapas mediante el método Delphi (Bravo y Arrieta, 2005):

1. Fase preliminar: se delimitó el contexto (limitaciones en las escalas de estilos parentales), el objetivo (diseñar un cuestionario de socialización parental relevante en la aparición y mantenimiento de los TC y los TRS) y se seleccionó un grupo de expertos nacionales (Valencia y Galicia) e internacionales (Uruguay, Venezuela, Argentina y USA), psiquiatras y psicólogos especialistas en trastornos infanto-juveniles, con experiencia acreditada a nivel asistencial, docente e investigador desde diferentes modelos teóricos (cognitivo, conductual, sistémico y dinámico).

2. Fase exploratoria: elaboración y aplicación de las encuestas. A partir de las principales escalas sobre socialización parental y su experiencia profesional, los expertos propusieron las prácticas parentales que consideraban relevantes para la aparición y mantenimiento de TC y TRS. Se elaboró un material de análisis inicial formado por un listado de conceptos y definiciones clave, con los cuales se creó una encuesta que los expertos debían cumplimentar indicando lo 
relevante del concepto y justificando dicha opinión. Tras varios ciclos de análisis de la encuesta retroalimentados con los resultados de la ronda anterior, se obtuvieron los conceptos que fueron finalmente recogidos en el cuestionario. Se decidió que para evaluar estos conceptos se usaría un cuestionario de categorías ordenadas con ítems respondidos mediante escala Likert de 5 puntos desde totalmente en desacuerdo hasta totalmente de acuerdo. A continuación, cada uno de los expertos propuso varios ítems para evaluar cada uno de los conceptos. Para la elaboración de los ítems debían seguir las siguientes recomendaciones: tiempo presente, relevantes, claros, un solo contenido, en modo directo e inverso y evitar el uso de negaciones (Abad, Olea, Ponsoda y García, 2011). Una vez recopilado este banco de ítems para cada uno de los conceptos, se encuestó de nuevo a los expertos pidiéndoles que seleccionaran el ítem que consideraban más adecuado para evaluar cada concepto y justificaran dicha selección. Tras tres ciclos de análisis de la encuesta retroalimentados con los resultados de la ronda anterior, se obtuvieron los ítems que fueron finalmente incluidos en el cuestionario.

3. Fase final: con los resultados obtenidos del análisis estadístico se seleccionaron los 38 principales conceptos relevantes y los 38 ítems más adecuados para evaluarlos, elaborando así el cuestionario.

Mediante este procedimiento se obtuvo la versión 1 del cuestionario.

\section{Muestra}

La muestra se compuso de 631 adolescentes de ambos géneros pertenecientes a centros públicos de educación secundaria de la Comunidad Valenciana (España) y sus cuidadores principales. Los adolescentes estaban cursando $3^{\circ}$ o $4^{\circ}$ de la ESO. Los criterios de inclusión eran: a) tener entre 14 y 16 años, b) convivencia en el seno familiar, c) aceptación voluntaria del adolescente y su familia de participar en el estudio mediante la firma del consentimiento informado. El criterio de exclusión fue ser adoptado. Se realizó un muestreo intencional de conveniencia ofreciendo participar en el estudio a todos los alumnos de $3^{\circ}$ y $4^{\circ}$ de la ESO de los once institutos que participaron en el estudio. Se ofreció la participación en el estudio a 706 adolescentes (10,62\% de rechazos). De los 631 cuidadores devolvieron cumplimentado el cuestionario 485 (23,13\% de abandonos).

\section{Instrumentos}

- Cuestionario de socialización parental TXP versión 1: consta de 38 ítems que evalúan 38 prácticas de socialización parental implicadas en la aparición y mantenimiento de TC y TRS. Diseñado con el método Delphi. Se responde con una escala Likert de 5 puntos desde totalmente en desacuerdo hasta totalmente de acuerdo.
Se considera que a mayor puntuación en el ítem y el cuestionario, menos probabilidad de que aparezcan TC y TRS. Se utilizaron dos versiones: una para el adolescente $(T X P-A)$ y otra para su cuidador principal $(T X P-C)$. El cuidador principal era la persona que más influía en su educación y pasaba más tiempo del día con él, según el propio adolescente. En TXP-A se incluyen 6 ítems inversos $(2,15,35,36,37,38)$ que fueron recodificados para la corrección. En TXP-C se incluyen 5 ítems inversos $(2,12,35,36,37)$.

- Entrevista estructurada para recogida de datos socio-familiares y antecedentes familiares de alcoholismo, drogodependencia y enfermedad mental.

- Entrevista estructurada para la evaluación de los criterios diagnósticos DSM-IV-TR para el trastorno disocial y el trastorno negativista desafiante (American Psychiatric Association, 2002).

- Cuestionario de Análisis Clínico (CAQ; Krug, 1994). Evalúa 12 escalas clínicas: hipocondría, depresión suicida, agitación, depresión ansiosa, depresión-baja energía, culpabilidad-resentimiento, apatía-retirada, paranoia, desviación psicopática, esquizofrenia, psicastenia y desajuste psicológico. Consta de 144 ítems de tres alternativas. La versión española muestra un valor medio del coeficiente alfa satisfactorio y validez discriminante moderada (Forns, Amador, Abad y Martorell, 1998).

- Cuestionario de personalidad para adolescentes (HSPQ, Cattell y Cattell, 1981). Compuesto por 140 ítems con tres opciones de respuesta. Permite apreciar 18 dimensiones de la personalidad: ansiedad, extraversión, excitabilidad, independencia, reservado-abierto, inteligencia, estabilidad, calma-excitabilidad, sumisión-dominancia, entusiasmo, despreocupación, emprendedor, sensibilidad, seguridad, serenidad, sociabilidad, integración, relajación. Los índices de consistencia interna de las escalas oscilan entre 0,66 y 0,86 y los de fiabilidad test-retest entre 0,69 y 0,87 (Cattell y Cattell, 1995).

- Cuestionario de tamizaje de los problemas en los adolescentes (POSIT; National Institute on Drug Abuse, 1991). Se usó la versión abreviada para la evaluación del uso/ abuso de drogas compuesta por 19 ítems con dos alternativas de respuesta (síno). A más puntuación, menos problemas con las drogas y el alcohol, usándose como punto de corte para considerar que el sujeto tiene problemas significativos una puntuación igual o menor a 33. La versión española presenta elevadas consistencia interna (alfa=0,82), sensibilidad $(94,3 \%)$ y especificidad 83,9\%) (Araujo, Golpe, Braña, Varela y Rial, 2018).

\section{Procedimiento}

Se obtuvieron los permisos de la Consellería de Educación de la Generalitat Valenciana y del comité ético de investigación del Consorcio Hospitalario Provincial de Cas- 
tellón (11 abril de 2008). Se siguieron los principios de la Declaración de Helsinki. Se contactó con los centros educativos para ofrecerles la posibilidad de participar en el estudio. Un psicólogo acudía a aquellos centros que aceptaron colaborar y solicitaba la participación voluntaria y desinteresada de los adolescentes y sus familias, quienes aceptaban participar firmando un consentimiento informado.

Una vez aceptada la participación, un psicólogo realizaba la evaluación en dos sesiones. Ambas sesiones se realizaban en horario lectivo en el propio centro escolar, aprovechando siempre que era posible el horario de tutoría. En la primera se realizaba una entrevista con el adolescente para la obtención de los datos sociodemográficos, identificar al cuidador principal (la persona que según el adolescente más influía en su educación y pasaba más tiempo del día con él) y evaluar los antecedentes familiares y los criterios diagnósticos de trastorno de conducta disocial y trastorno negativista desafiante. También se administraban el $C A Q$ y el TXP-A y se le daba al adolescente el TXP-C para el cuidador principal que debía devolver cumplimentado en la siguiente sesión. Al cabo de quince días, en la segunda sesión, se recogía el TXP-C y se administraban al adolescente el $H S P Q$, el POSIT y de nuevo el TXP-A. También se entregaba de nuevo el TXP-C para que el adolescente lo devolviera cumplimentado por el cuidador principal.

\section{Análisis de datos}

Se usaron los programas SPSS v.20 (IBM Corp. Released, 2011), Factor 10.5.03 (Lorenzo-Seva y Ferrando, 2006) para los análisis factoriales y Jmetrik 4.0.5 (Meyer, 2014) para el análisis de ítems desde la teoría clásica de los tests y la teoría de respuesta al ítem. El estudio descriptivo de las variables mostró que los datos de los cuestionarios presentaban curtosis. El porcentaje de valores perdidos fue menor del $5 \%$, estos valores se eliminaban, según permitiera el procedimiento, por pareja o por casos. Se realizó la validación psicométrica del TXP-A. Se dividió la muestra en dos mitades aleatoriamente. Se recodificaron los ítems inversos. Para averiguar si era conveniente realizar un análisis factorial del cuestionario se usaron el índice Kaiser-Meyer-Olkin (KMO) y la prueba de Esferidad de Barlett. En una mitad de la muestra se efectuó el análisis factorial exploratorio de la versión 1 del cuestionario a partir de las correlaciones policóricas con extracción de mínimos cuadrados no ponderados y se utilizaron como reglas de extracción de factores el análisis paralelo y el Scree-test de Catell. El método de rotación utilizado fue Promin. Se eliminaron los ítems que saturaban menos de 0,35 en los factores obtenidos, creando así la versión 2 del cuestionario. Con la otra mitad de la muestra se realizó un análisis factorial confirmatorio de la versión 2 a partir de la matriz de correlaciones policóricas. Se calcularon el índice RMSR (aceptables los valores comprendidos entre 0,05 y 0,08 ), el índice de bondad de ajuste (GFI; valores mayores de 0,9 indican buen ajuste), el índice
RMSEA (aceptables los valores comprendidos entre 0,05 y $0,08)$ y el ajuste mínimo ( $>0,05$ indica bondad de ajuste) para valorar el ajuste de la solución factorial obtenida (Hair, Anderson, Tatham y Black, 1998). Se eliminaron los ítems que saturaban menos de 0,35 en los factores obtenidos, creando así la versión 3 del cuestionario. Se realizó de la misma forma el análisis factorial exploratorio y confirmatorio del TXP-C, obteniendo la versión 3 del cuestionario. En la muestra total se obtuvieron las saturaciones factoriales de los ítems de las versiones definitivas, el índice RMSR, el GFI, el índice RMSEA y el ajuste mínimo . También se realizó el análisis de ítems desde la teoría clásica de los test y desde la teoría de respuesta al ítem, incluyendo análisis de Rasch y funcionamiento diferencial con el procedimiento Mantel-Haenszel. Se calcularon los baremos percentiles para ambas versiones del cuestionario. Para evaluar la fiabilidad de las puntuaciones se usó alfa de Cronbach y el mayor límite inferior (GLB). Se usó el coeficiente de correlación de Pearson para evaluar validez convergente y discriminante y fiabilidad interjueces, coeficiente de correlación intraclase para la fiabilidad test-restest y para analizar posible funcionamiento diferencial se usó coeficiente de correlación de Pearson, prueba ty ANOVA. Se calculó el tamaño del efecto (TE) de la prueba $t$ mediante $d$ de Cohen $(0,2$ efecto pequeño, 0,5 mediano y 0,8 grande) y el de ANOVA mediante eta cuadrado parcial $(0,01$ efecto pequeño, 0,06 mediano y 0,14 grande).

\section{Resultados}

\section{Datos sociodemográficos y psicopatológicos de la muestra}

El 42,8\% (n= 269) de la muestra eran varones y el 57,2\% $(\mathrm{n}=359)$ mujeres. La media de edad era 15,27 años (DT= $0,70)$, con un rango entre 14 y 16 años. El 58\% cursaba $3^{\circ}$ de la E.S.O. y el $42 \%$ cursaba $4^{\circ}$ de la E.S.O. El $85,1 \%$ tenía nacionalidad española, el 9,4\% provenía de otros países europeos, el 3,6\% de Centro y Sudamérica, el 1,3\% de África y el $0,6 \%$ tenía otras nacionalidades.

El 15,6\% de los adolescentes eran hijos únicos, el 1,9\% tenían un hermano y el $82,5 \%$ dos o más hermanos. El $78,9 \%$ de los padres de los adolescentes estaban casados incluyendo pareja de hecho, el 7,1\% estaban casados en segundas nupcias, el 4,7\% separados, el 6,6\% divorciados, el 2,1\% eran viudos y el 0,5\% solteros. En el 90,7\% de los casos el cuidador principal era la madre, en el 7,3\% el padre y en el $2 \%$ fueron los abuelos, tíos, hermana o la pareja del padre o madre.

El 13,3\% de los adolescentes presentaban antecedentes familiares de alcoholismo, el 8,4\% tenía algún familiar con problemas de drogodependencia y el $13,9 \%$ presentaban antecedentes familiares de enfermedad mental. El 0,6\% $(\mathrm{n}=4)$ cumplía criterios de trastorno disocial y el $0,6 \%(\mathrm{n}=$ 4) de trastorno negativista desafiante. En la tabla 1 se pue- 
den observar las puntuaciones en el $C A Q$ y el $H S P Q$. La media en el POSIT fue 36,56 (DT= 2,07), encontrándose que el $7,4 \%(n=46)$ de la muestra presentaba problemas con las drogas o el alcohol.

\section{Análisis de los ítems versión 1 (n adolescentes $=316$ y $n$ cuidadores $=$ 235)}

En la versión 1 del TXP-A, el rango de puntuaciones medias de los ítems osciló entre 2,72 y 4,66. El análisis descriptivo de los ítems puede verse en la tabla 2. El ítem que puntuó más alto fue el 37 y el que menor puntuación obtuvo fue el 35. Se obtuvo una consistencia interna para el total de la escala de 0,89. El rango de correlaciones de cada uno de los ítems con la puntuación total de la escala corregida osciló entre -0,009 (ítem 1) y 0,71 (ítems 17 y 18).

En la versión 1 del $T X P$ - $C$, el rango de puntuaciones medias de los ítems osciló entre 2,17 y 4,97. El análisis descriptivo de los ítems puede verse en la tabla 2. El ítem que puntuó más alto fue el 24 y el que menor puntuación obtuvo fue el 35. Se obtuvo una consistencia interna para el total de la escala de 0,80. El rango de correlaciones de cada uno de los ítems con la puntuación total de la escala corregida osciló entre -0,17 (ítem 12) y 0,63 (ítem 17 ).

\section{Análisis factorial exploratorio versión 1 TXP-A $(n=316)$}

El índice KMO fue 0,90 y la prueba de esfericidad de Bartlett $(703)=4367,009 ; \mathrm{p}<0,001$. El análisis paralelo y el Scree-test de Catell recomendaron una estructura de dos factores. Ambos factores presentaban una correlación de 0,74 . Se eliminaron los ítems $3,11,15,16,32$ y 36 por presentar saturaciones menores de 0,35 , creando una versión 2 de 32 ítems. En la tabla 2 se pueden ver las saturaciones factoriales de cada ítem.

\section{Análisis factorial confirmatorio versión 2 TXP-A $(n=315)$}

El índice KMO fue 0,90 y la prueba de esfericidad de Bartlett $(496)=3842,3 ; \mathrm{p}<0,001$. Se eliminaron los ítems 7, 10 y 31 por presentar saturaciones menores de 0,35 , creando una versión 3 de 29 ítems. Para el factor 1 el valor propio fue de 11,42 y el porcentaje de varianza explicada $35,6 \%$; para el factor 2 el valor propio fue 2,65 y el porcentaje de varianza explicada $8,3 \%$. La varianza explicada total fue $43,9 \%$. La correlación entre ambos factores fue 0,72. El RMSR fue 0,05, el GFI 0,97, el RMSEA 0,03 y el ajuste mínimo $(433)=546331(\mathrm{p}<0,001)$. En la tabla 2 se pueden ver las saturaciones factoriales de cada ítem.

\section{Análisis factorial exploratorio versión 1 TXP-C $(n=235)$}

El índice KMO fue 0,79 y la prueba de esfericidad de Bartlett $(703)=2475,547 ; \mathrm{p}<0,001$. El análisis paralelo y el Scree-test de Catell recomendaron una estructura de dos factores. Se eliminaron los ítems 1, 2, 3, 4, 7, 8, 9, 10, 11, $12,15,18,19,20,30,31,33,35,36,37$ y 38 por presentar saturaciones menores de 0,35 , creando una versión 2 de 17 ítems. En la tabla 2 se pueden ver las saturaciones factoriales de cada ítem.

Tabla 1. Puntuaciones medias de los adolescentes en el Cuestionario de Análisis Clínico (CAQ) de Krug y el Cuestionario de personalidad para adolescentes (HSPQ) de Cattell y Cattell.

\begin{tabular}{|c|c|c|c|}
\hline \multicolumn{2}{|c|}{ Cuestionario Análisis Clínico CAQ } & \multicolumn{2}{|c|}{ Cuestionario personalidad adolescentes HSPQ } \\
\hline Dimensión & Media (DT) & Dimensión & Media (DT) \\
\hline Hipocondriasis & $5,78(1,81)$ & Ansiedad & $1,55(2,90)$ \\
\hline Depresión suicida & $5,89(1,73)$ & Extraversión & $7,04(2,93)$ \\
\hline Agitación & $5,92(1,84)$ & Excitabilidad & $11,43(2,18)$ \\
\hline Depresión ansiosa & $5,69(1,76)$ & Independencia & $7,03(2,93)$ \\
\hline Depresión baja energía & $5,78(1,92)$ & Reservado-Abierto & $5,86(1,84)$ \\
\hline Culpa-resentimiento & $5,15(1,95)$ & Inteligencia & $4,36(1,92)$ \\
\hline Apatía- retirada & $5,23(1,84)$ & Estabilidad & $6,23(2,00)$ \\
\hline Paranoia & $5,86(1,81)$ & Calma-Excitabilidad & $4,61(1,77)$ \\
\hline Desviación Psicopática & $5,75(1,78)$ & Sumisión-Dominancia & $6,48(1,74)$ \\
\hline Esquizofrenia & $5,35(1,77)$ & Entusiasmo & $5,37(1,89)$ \\
\hline Psicastenia & 4,97 (1,91) & Despreocupación & $5,56(1,77)$ \\
\hline \multirow[t]{7}{*}{ Desajuste psicológico } & $5,17(1,82)$ & Emprendedor & $6,51(1,77)$ \\
\hline & & Sensibilidad & $5,83(1,75)$ \\
\hline & & Seguridad & $6,26(1,71)$ \\
\hline & & Serenidad & $4,68(1,90)$ \\
\hline & & Sociabilidad & $5,83(1,84)$ \\
\hline & & Integración & $5,94(1,94)$ \\
\hline & & Relajación & $4,31(1,77)$ \\
\hline
\end{tabular}


Ana Benito, Gema Calvo, Matías Real-López, María José Gallego, Sonia Francés, Ángel Turbi, Gonzalo Haro

Tabla 2. Análisis de los ítems y saturaciones factoriales en el análisis factorial exploratorio de la versión 1 y saturaciones factoriales en el análisis factorial confirmatorio de la versión 2 del cuestionario para adolescentes y el cuestionario para cuidadores.

\begin{tabular}{|c|c|c|c|c|c|c|c|c|c|c|}
\hline \multirow[t]{2}{*}{ ÍTEM } & \multicolumn{4}{|c|}{ ADOLESCENTES } & \multicolumn{6}{|c|}{ CUIDADORES } \\
\hline & M & DT & RC & $\begin{array}{c}\text { SFE } \\
(n=316)\end{array}$ & $\begin{array}{c}\text { SFC } \\
(n=315)\end{array}$ & $M$ & DT & RC & $\begin{array}{c}\text { SFE } \\
(n=235)\end{array}$ & $\begin{array}{c}\text { SFC } \\
(n=250)\end{array}$ \\
\hline $\begin{array}{l}\text { 3. Mis padres conocen y controlan todas mis actividades } \\
\text { y amistades/Suelo conocer y controlar todas las } \\
\text { actividades y amistades de mi hijo. }\end{array}$ & 3,25 & 1,29 & 0,23 & $F 1=0,33$ & $E$ & 4,06 & 1,00 & 0,13 & $\mathrm{~F} 2=0,03$ & $E$ \\
\hline $\begin{array}{l}\text { 4. Mis padres me dejan participar en la creación de las } \\
\text { normas/Mis hijos participan en la elaboración de las } \\
\text { normas familiares. }\end{array}$ & 3,44 & 1,19 & 0,34 & $F 2=0,62$ & $F 1=0,44$ & 3,83 & 1,18 & 0,24 & $\mathrm{~F} 1=0,13$ & $E$ \\
\hline $\begin{array}{l}\text { 5. En mi familia todos nos sentimos muy cercanos y nos } \\
\text { mantenemos unidos y fieles unos a otros }\end{array}$ & 4,07 & 1,05 & 0,63 & $\mathrm{~F} 2=0,56$ & $F 1=0,63$ & 4,48 & 0,83 & 0,55 & $F 1=0,66$ & $F 2=0,77$ \\
\hline $\begin{array}{l}\text { 6. Considero que mis padres son accesibles y están } \\
\text { dispuestos a ayudar/Creo que en nuestra familia somos } \\
\text { accesibles y estamos a disposición los unos de los otros. }\end{array}$ & 4,42 & 0,87 & 0,57 & $F 2=0,54$ & $F 1=0,53$ & 4,59 & 0,78 & 0,52 & $F 1=0,59$ & $F 2=0,51$ \\
\hline $\begin{array}{l}\text { 7. En mi familia a menudo realizamos tareas y } \\
\text { actividades en común. }\end{array}$ & 3,36 & 1,13 & 0,53 & $F 1=0,35$ & $F 1=0,34$ & 4,03 & 1,07 & 0,42 & $\mathrm{~F} 1=0,34$ & $E$ \\
\hline $\begin{array}{l}\text { 8. En nuestra familia están diferenciados de forma clara } \\
\text { los papeles, tareas y responsabilidades propios de los } \\
\text { padres y los hijos, manteniéndose los límites entre éstos. }\end{array}$ & 3,46 & 1,13 & 0,08 & $\mathrm{~F} 1=0,65$ & $F 2=0,71$ & 3,79 & 1,15 & 0,22 & $F 2=0,11$ & $E$ \\
\hline $\begin{array}{l}\text { 9. Las normas en mi casa se cumplen de forma habitual } \\
\text { y cuando se incumplen mis padres aplican medidas } \\
\text { correctoras/Las normas en nuestra casa se cumplen } \\
\text { de forma habitual y cuando se incumplen los padres } \\
\text { aplicamos medidas correctoras. }\end{array}$ & 3,62 & 1,09 & 0,23 & $F 1=1,009$ & $F 2=0,97$ & 3,78 & 1,20 & 0,22 & $F 2=0,12$ & $E$ \\
\hline $\begin{array}{l}\text { 11. Mi familia me ha enseñado que puedo confiar en } \\
\text { los demás: el mundo es un lugar seguro/Transmitimos a } \\
\text { nuestros hijos confianza en los demás: el mundo es un } \\
\text { lugar seguro. }\end{array}$ & 2,81 & 1,11 & 0,16 & $F 2=0,23$ & $E$ & 2,83 & 1,29 & 0,23 & $F 1=0,07$ & $E$ \\
\hline $\begin{array}{l}\text { 12. Mis padres favorecen las relaciones con otras } \\
\text { personas de fuera de la familia, me animan a que haga } \\
\text { actividades fuera de casa y aceptan que traiga amigos a } \\
\text { casa/Nos gusta más estar juntos que con personas de } \\
\text { fuera de la familia. }\end{array}$ & 4,26 & 0,94 & 0,47 & $F 2=0,62$ & $F 1=0,51$ & $\begin{array}{l}2,58 \\
(I)\end{array}$ & 1,26 & $-0,17$ & $F 2=0,01$ & $E$ \\
\hline $\begin{array}{l}\text { 13. En mi familia le damos importancia a valores } \\
\text { sociales como el respeto, la solidaridad, la tolerancia, } \\
\text { etc./Educamos a nuestros hijos en valores como el } \\
\text { respeto, la solidaridad, la tolerancia, etc. }\end{array}$ & 4,40 & 0,86 & 0,51 & $F 1=0,51$ & $F 2=0,37$ & 4,81 & 0,57 & 0,26 & $F 2=0,58$ & $F 1=0,67$ \\
\hline $\begin{array}{l}\text { 14. Mis padres cumplen su papel como padres y se } \\
\text { sienten a gusto haciéndolo/Los padres estamos a gusto } \\
\text { siéndolo y asumimos nuestro papel. }\end{array}$ & 4,55 & 0,75 & 0,57 & $F 2=0,48$ & $F 2=0,50$ & 4,68 & 0,60 & 0,45 & $F 1=0,40$ & $F 1=0,44$ \\
\hline $\begin{array}{l}\text { 15. Mis padres me siguen tratando ahora igual que } \\
\text { cuando era un niño/A medida que la familia y las } \\
\text { circunstancias cambian, voy cambiando mi relación con } \\
\text { los hijos y nos adaptamos a los cambios. }\end{array}$ & $\begin{array}{l}3,58 \\
(I)\end{array}$ & 1,30 & 0,17 & $F 2=0,16$ & $E$ & 4,40 & 0,81 & 0,40 & $F 2=0,28$ & $E$ \\
\hline $\begin{array}{l}\text { 16. En mi familia expresamos nuestras emociones } \\
\text { frecuente e intensamente. }\end{array}$ & 3,32 & 1,04 & 0,43 & $F 1=0,32$ & $E$ & 4,16 & 0,99 & 0,38 & $F 2=0,45$ & $\mathrm{~F} 1=0,31$ \\
\hline $\begin{array}{l}\text { 17. En nuestra casa se vive un ambiente familiar cálido } \\
\text { y positivo. }\end{array}$ & 4,03 & 1,03 & 0,71 & $F 2=0,66$ & $F 1=0,62$ & 4,42 & 0,82 & 0,63 & $\mathrm{~F} 1=0,77$ & $F 2=0,81$ \\
\hline $\begin{array}{l}\text { 18. En mi familia nos comprendemos unos a otros/Como } \\
\text { padres intentamos comprender a nuestros hijos. }\end{array}$ & 3,77 & 1,09 & 0,71 & $F 2=0,63$ & $\mathrm{~F} 1=0,72$ & 4,82 & 0,52 & 0,40 & $\mathrm{~F} 1=0,33$ & $E$ \\
\hline
\end{tabular}


19. Me han educado para que asuma la responsabilidad de mis acciones y sus consecuencias/En nuestra familia cada uno asumimos la responsabilidad de nuestras acciones y sus consecuencias.

\begin{tabular}{|c|c|c|c|c|c|c|c|c|c|c|}
\hline $\begin{array}{l}\text { 20. Mis padres me apoyan emocionalmente/Cuando } \\
\text { tengo problemas me apoyo emocionalmente en mis hijos. }\end{array}$ & 4,26 & 0,92 & 0,68 & $F 2=0,60$ & $F 1=0,70$ & 3,30 & 1,36 & 0,14 & $F 2=0,09$ & $E$ \\
\hline $\begin{array}{l}\text { 21. En mi familia sabemos resolver los problemas sin } \\
\text { demasiada tensión. }\end{array}$ & 3,37 & 1,13 & 0,61 & $F 2=0,63$ & $F 1=0,47$ & 3,57 & 1,13 & 0,47 & $F 1=0,56$ & $F 2=0,73$ \\
\hline $\begin{array}{l}\text { 22. En mi familia podemos expresar sin problemas todo } \\
\text { tipo de sentimientos: alegría, tristeza, cariño, miedo, } \\
\text { rabia, etc. }\end{array}$ & 4,00 & 1,05 & 0,62 & $F 2=: 0,49$ & $F 1=0,50$ & 4,55 & 0,73 & 0,32 & $F 2=0,64$ & $F 1=0,38$ \\
\hline $\begin{array}{l}\text { 23. En mi familia nos expresamos y nos entendemos con } \\
\text { claridad. }\end{array}$ & 3,77 & 1,04 & 0,64 & $F 2=0,54$ & $F 1=0,55$ & 4,24 & 0,91 & 0,62 & $F 1=0,65$ & $F 2=0,80$ \\
\hline $\begin{array}{l}\text { 24. Me siento importante para mis padres y valorado } \\
\text { por ellos/Valoro a mis hijos como parte importante de } \\
\text { la familia. }\end{array}$ & 4,31 & 0,96 & 0,65 & $F 2=0,78$ & $F 1=0,69$ & 4,97 & 0,25 & 0,23 & $F 2=0,68$ & $F 1=1,01$ \\
\hline $\begin{array}{l}\text { 25. En mi familia me tratan con cariño/En nuestra familia } \\
\text { nos damos cariño habitualmente. }\end{array}$ & 4,56 & 0,75 & 0,68 & $F 2=0,88$ & $F 1=0,77$ & 4,67 & 0,69 & 0,60 & $F 1=0,56$ & $F 2=0,63$ \\
\hline 26. Mis padres (o los que me educan en casa) están & 4,42 & 0,75 & 0,46 & $F 1=0,37$ & $F 2=0,53$ & 3,90 & 1,12 & 0,39 & $F 1=0,56$ & $F 2=0,65$ \\
\hline
\end{tabular}

de acuerdo en la forma de educarme/Mi pareja y yo estamos de acuerdo y actuamos de forma conjunta, sin contradecirnos, en las tareas de crianza y educación de los hijos (en caso de compartir la educación de los hijos con otra persona diferente de la pareja referirse a ella).

27. Mis padres me permiten, enseñan y favorecen que me relacione con mis amigos y otras personas/Enseño y favorezco que mis hijos se relacionen con la gente de forma adecuada.

28. Mis padres respetan mis derechos y mi intimidad/ En mi familia nos respetamos y tenemos en cuenta la intimidad e individualidad de cada uno de nosotros.

$\begin{array}{llllllllll}4,55 & 0,60 & 0,31 & F 1=0,55 & F 2=0,51 & 4,30 & 0,87 & 0,23 & F 2=0,21 & E\end{array}$

(1)

$4,550,60$


Ana Benito, Gema Calvo, Matías Real-López, María José Gallego, Sonia Francés, Ángel Turbi, Gonzalo Haro

Tabla 3. Análisis de los ítems, saturaciones factoriales y correlación con la escala corregida de la versión 3 del cuestionario para adolescentes ( $n=631$ ) y el cuestionario para cuidadores $(n=485)$.

\begin{tabular}{|c|c|c|c|c|c|c|c|c|c|}
\hline \multirow{28}{*}{$\begin{array}{l}\text { CUESTIONARIO } \\
\text { ADOLESCENTES } \\
\text { VERSIÓN } 3\end{array}$} & ÍTEM & $M$ & DT & IDis & RC & SF & IC(95\%)SF & WMS & UMS \\
\hline & 1 & 3,09 & 1,15 & 0,04 & 0,04 & $F 2=0,81$ & $0,68-0,96$ & 1,54 & 1,73 \\
\hline & 2 & 3,50 & 1,28 & 0,12 & 0,12 & $F 1=0,73$ & $0,64-0,87$ & 1,77 & 1,96 \\
\hline & 4 & 3,41 & 1,19 & 0,29 & 0,29 & $F 1=0,53$ & $0,38-0,67$ & 1,27 & 1,43 \\
\hline & 5 & 4,06 & 1,07 & 0,63 & 0,63 & $\mathrm{~F} 1=0,58$ & $0,45-0,68$ & 0,89 & 0,83 \\
\hline & 6 & 4,41 & 0,87 & 0,54 & 0,54 & $F 1=0,51$ & $0,38-0,63$ & 1,01 & 0,95 \\
\hline & 8 & 3,39 & 1,14 & 0,11 & 0,11 & $\mathrm{~F} 2=0,70$ & $0,59-0,84$ & 1,42 & 1,54 \\
\hline & 9 & 3,53 & 1,15 & 0,24 & 0,24 & $F 2=0,99$ & $0,90-1,11$ & 1,29 & 1,35 \\
\hline & 12 & 4,25 & 0,99 & 0,46 & 0,46 & $F 1=0,52$ & $0,36-0,67$ & 1,14 & 1,11 \\
\hline & 13 & 4,41 & 0,82 & 0,49 & 0,49 & $F 2=0,46$ & $0,31-0,59$ & 0,97 & 0,88 \\
\hline & 14 & 4,52 & 0,75 & 0,58 & 0,58 & $\mathrm{~F} 2=0,40$ & $0,27-0,52$ & 0,85 & 0,76 \\
\hline & 17 & 4,01 & 1,01 & 0,69 & 0,69 & $F 1=0,63$ & $0,50-0,73$ & 0,67 & 0,67 \\
\hline & 18 & 3,80 & 1,05 & 0,67 & 0,67 & $\mathrm{~F} 1=0,66$ & $0,56-0,78$ & 0,65 & 0,66 \\
\hline & 19 & 4,51 & 0,68 & 0,36 & 0,36 & $F 2=0,59$ & $0,45-0,74$ & 0,94 & 0,91 \\
\hline & 20 & 4,20 & 0,92 & 0,65 & 0,65 & $F 1=0,62$ & $0,52-0,71$ & 0,74 & 0,68 \\
\hline & 21 & 3,32 & 1,14 & 0,62 & 0,62 & $\mathrm{~F} 1=0,51$ & $0,40-0,63$ & 0,69 & 0,69 \\
\hline & 22 & 3,96 & 1,09 & 0,61 & 0,61 & $\mathrm{~F} 1=0,46$ & $0,35-0,55$ & 0,88 & 0,84 \\
\hline & 23 & 3,79 & 1,03 & 0,63 & 0,63 & $F 1=0,52$ & $0,37-0,62$ & 0,68 & 0,68 \\
\hline & 24 & 4,35 & 0,93 & 0,62 & 0,62 & $F 1=0,72$ & $0,61-0,82$ & 0,94 & 0,80 \\
\hline & 25 & 4,54 & 0,75 & 0,66 & 0,66 & $\mathrm{~F} 1=0,80$ & $0,69-0,90$ & 0,81 & 0,66 \\
\hline & 26 & 4,41 & 0,77 & 0,52 & 0,53 & $F 2=0,52$ & $0,37-0,63$ & 0,81 & 0,81 \\
\hline & 27 & 4,47 & 1,77 & 0,57 & 0,57 & $F 1=0,48$ & $0,35-0,65$ & 0,84 & 0,73 \\
\hline & 28 & 4,09 & 1,02 & 0,51 & 0,51 & $\mathrm{~F} 1=0,64$ & $0,52-0,77$ & 1,00 & 0,92 \\
\hline & 33 & 3,87 & 0,95 & 0,50 & 0,50 & $F 2=0,79$ & $0,69-0,90$ & 0,77 & 0,76 \\
\hline & 34 & 4,12 & 0,94 & 0,65 & 0,65 & $F 1=0,62$ & $0,51-0,72$ & 0,68 & 0,75 \\
\hline & 35 & 2,76 & 1,25 & 0,11 & 0,11 & $\mathrm{~F} 1=0,40$ & $0,27-0,55$ & 1,63 & 1,78 \\
\hline & 37 & 4,65 & 0,77 & 0,27 & 0,27 & $\mathrm{~F} 1=0,53$ & $0,37-0,68$ & 1,68 & 1,58 \\
\hline & 38 & 4,58 & 0,93 & 0,47 & 0,47 & $F 1=0,58$ & $0,41-0,72$ & 1,73 & 1,59 \\
\hline \multirow{16}{*}{$\begin{array}{l}\text { CUESTIONARIO } \\
\text { CUIDADORES } \\
\text { VERSIÓN } 3\end{array}$} & 5 & 4,44 & 0,85 & 0,59 & 0,63 & $F 2=0,67$ & $0,42-0,87$ & 0,89 & 0,80 \\
\hline & 6 & 4,61 & 0,78 & 0,54 & 0,56 & $F 2=0,57$ & $0,37-0,78$ & 1,29 & 0,99 \\
\hline & 13 & 4,82 & 0,57 & 0,29 & 0,27 & $F 1=0,81$ & $0,63-1,17$ & 1,82 & 1,61 \\
\hline & 14 & 4,72 & 0,63 & 0,48 & 0,49 & $F 2=0,41$ & $0,15-0,68$ & 1,20 & 1,06 \\
\hline & 17 & 4,44 & 0,85 & 0,70 & 0,71 & $F 2=0,77$ & $0,61-0,95$ & 0,72 & 0,67 \\
\hline & 21 & 3,59 & 1,14 & 0,51 & 0,52 & $F 2=0,76$ & $0,59-0,97$ & 1,07 & 1,14 \\
\hline & 22 & 4,53 & 0,76 & 0,39 & 0,34 & $\mathrm{~F} 1=0,73$ & $0,45-1,007$ & 1,30 & 1,56 \\
\hline & 23 & 4,23 & 0,91 & 0,59 & 0,63 & $F 2=0,72$ & $0,57-0,87$ & 0,83 & 0,85 \\
\hline & 24 & 4,96 & 0,25 & 0,40 & 0,41 & $F 1=0,87$ & $0,66-1,05$ & 1,01 & 0,43 \\
\hline & 25 & 4,62 & 0,69 & 0,60 & 0,65 & $F 2=0,59$ & $0,43-0,74$ & 0,91 & 0,80 \\
\hline & 26 & 3,97 & 1,30 & 0,47 & 0,49 & $F 2=0,85$ & $0,60-1,18$ & 1,34 & 1,33 \\
\hline & 27 & 4,86 & 0,43 & 0,45 & 0,44 & $F 1=0,88$ & $0,68-1,15$ & 1,07 & 0,80 \\
\hline & 28 & 4,57 & 0,73 & 0,52 & 0,55 & $F 2=0,64$ & $0,38-0,83$ & 1,06 & 0,99 \\
\hline & 29 & 4,45 & 0,80 & 0,58 & 0,59 & $F 2=0,63$ & $0,48-0,84$ & 0,89 & 0,85 \\
\hline & 32 & 4,61 & 1,48 & 0,28 & 0,46 & $F 2=0,75$ & $0,52-1,002$ & 1,45 & 1,38 \\
\hline & 34 & 4,48 & 0,71 & 0,61 & 0,64 & $F 2=0,77$ & $0,65-0,94$ & 0,70 & 0,79 \\
\hline
\end{tabular}

Nota. M: Media; DT: Desviación típica; IDis: Índice de Discriminación; RC: correlación de cada ítem de la escala con la puntuación de ésta corregida, SF: saturación factorial en el análisis factorial confirmatorio de la versión 3; F1: el ítem satura en el factor 1; F2: el ítem satura en el factor 2; IC(5\%)SF: Intervalo de confianza 95\% de la saturación factorial; WMS: Media residuos cuadrados ponderada (Infit); UMS: Media residuos cuadrados no ponderada (Outfit). 
Análisis factorial confirmatorio versión 2 TXP-C $(n=250)$

El índice KMO fue 0,89 y la prueba de esfericidad de Bartlett $(136)=1611,0 ; \mathrm{p}<0,001$. Se eliminó el ítem 16 por presentar saturación menor de 0,35 , creando una versión 3 de 16 ítems. Para el factor 1 el valor propio fue de 6,65 y el porcentaje de varianza explicada $39,1 \%$; para el factor 2 el valor propio fue 1,66 y el porcentaje de varianza explicada $9,7 \%$. La varianza explicada total fue 48,9\%. La correlación entre ambos factores fue 0,67. El RMSR fue 0,06, el GFI 0,98, el RMSEA 0,05 y el ajuste mínimo $(103)=112812(p<0,23)$. En la tabla 2 se pueden ver las saturaciones factoriales de cada ítem.

\section{Análisis de los ítems versión 3 (n adolescentes= 631 y n cuidadores $=$ 485)}

En la tabla 3 se pueden observar los resultados del análisis de los ítems y las saturaciones factoriales de la versión 3 del TXP-A. Para el factor 1 el valor propio fue de 10,83 y el porcentaje de varianza explicada $37,3 \%$; para el factor 2 el valor propio fue 2,63 y el porcentaje de varianza explicada $9,09 \%$. La varianza explicada total fue $46,4 \%$. La correlación entre ambos factores fue 0,73. El RMSR fue 0,04, el GFI 0,98, el RMSEA 0,03 y el ajuste mínimo $(349)=608468(\mathrm{p}<0,001)$.

En la tabla 3 se pueden ver los resultados del análisis de los ítems y las saturaciones factoriales de la versión 3 del TXP-C. Para el factor 1 el valor propio fue de 8,07 y el porcentaje de varianza explicada 50,4\%; para el factor 2 el valor propio fue 1,60 y el porcentaje de varianza explicada $10,04 \%$. La varianza explicada total fue $60,4 \%$. La correlación entre ambos factores fue 0,71. El RMSR fue 0,05, el GFI 0,98, el RMSEA $>0,1$ y el ajuste mínimo $(89)=170783(p<0,001)$.

Esta versión 3 se aceptó como la versión final del cuestionario. La versión final del TXP-A consta de 29 ítems. En el factor 1 saturan 20 ítems: 2, 4, 5, 6, 12, 17, 18, 20, 21, 22, $23,24,25,27,28,29,34,35,37$ y 38 . En el factor 2 saturan 9 ítems: 1, 8, 9, 13, 14, 19, 26, 30 y 33. La versión final del TXP-C consta de 16 ítems. En el factor 1 saturan 4 ítems: 13, 22, 24 y 27. En el factor 2 saturan 12 ítems: 5, 6, 14, 17, $21,23,25,26,28,29,32$ у 34 .

\section{Puntuaciones en el cuestionario}

La media para el TXP-A fue 80,45 (DT=11,84) en el factor $1 ; 35,28(\mathrm{DT}=4,79)$ en el factor 2 y $115,74(\mathrm{DT}=14,42)$ en el total. La media para el TXP-C fue 19,14 (DT=1,40) en el factor 1; 52,79 (DT=6,66) en el factor 2 y 71,97 (DT= 7,38 ) en el total. Los baremos percentiles de adolescentes y cuidadores pueden verse en la tabla 4 .

\section{Fiabilidad de las puntuaciones}

El cuestionario TXP-A presenta GLB $=0,97$ y un alfa de Cronbach de 0,89 , el factor 1 de 0,89 y el factor 2 de 0,71 .

Tabla 4. Baremos percentiles para las puntuaciones en los factores 1 y 2 y puntuación total del cuestionario de estilo de socialización parental para adolescentes y cuidadores.

\begin{tabular}{|c|c|c|c|c|c|c|}
\hline \multirow[b]{2}{*}{ PERCENTIL } & \multicolumn{3}{|c|}{ ADOLESCENTES } & \multicolumn{3}{|c|}{ CUIDADORES } \\
\hline & FACTOR 1 & FACTOR 2 & TOTAL & FACTOR 1 & FACTOR 2 & TOTAL \\
\hline 1 & 42,96 & 23,32 & 68,32 & 14 & 32 & 49,43 \\
\hline 5 & 59 & 27 & 90 & 16 & 40 & 57 \\
\hline 10 & 64,2 & 29 & 96 & 18 & 43,3 & 62 \\
\hline 15 & 69 & 30 & 100,8 & 18 & 47 & 65 \\
\hline 20 & 72 & 31 & 106 & 18 & 48 & 67 \\
\hline 25 & 74 & 32 & 109 & 19 & 50 & 68 \\
\hline 30 & 76 & 33 & 111 & 19 & 51 & 70 \\
\hline 35 & 78 & 34 & 113 & 19 & 52 & 71 \\
\hline 40 & 80 & 34 & 114 & 19 & 53 & 72 \\
\hline 45 & 81 & 35 & 116 & 20 & 54 & 73 \\
\hline 55 & 84 & 36 & 119,6 & 20 & 55 & 75 \\
\hline 60 & 85 & 37 & 121 & 20 & 56 & 76 \\
\hline 65 & 87 & 37 & 123 & 20 & 57 & 76 \\
\hline 70 & 88 & 38 & 125 & 20 & 57 & 77 \\
\hline 75 & 89 & 39 & 126 & 20 & 58 & 77 \\
\hline 80 & 90 & 40 & 128 & 20 & 58 & 78 \\
\hline 85 & 92 & 40 & 130 & 20 & 59 & 78 \\
\hline 90 & 94 & 42 & 133 & 20 & 59 & 79 \\
\hline 95 & 95 & 43 & 135 & 20 & 60 & 80 \\
\hline 99 & 98 & 45 & 138 & 20 & 60 & 80 \\
\hline
\end{tabular}


Tabla 5. Correlaciones entre las puntuaciones en el cuestionario de socialización parental de los adolescentes y sus cuidadores.

\begin{tabular}{llccl}
\hline & & \multicolumn{3}{c}{ ADOLESCENTE } \\
& & Puntuación total & Factor $\mathbf{1}$ & Factor 2 \\
\hline CUIDADOR & Puntuación total & $\mathbf{0 , 3 9 8 ^ { \star \star }}$ & $0,384^{\star \star}$ & $0,242^{\star \star}$ \\
& Factor 1 & $0,226^{\star \star}$ & $\underline{0,185^{\star \star}}$ & $\underline{0,221^{\star \star}}$ \\
& Factor 2 & $0,399^{\star \star}$ & $\underline{\mathbf{0 , 3 9 1 * *}}$ & $\underline{0,228^{\star \star}}$ \\
\hline
\end{tabular}

Nota. ${ }^{\star *}$ p $<0,01$. En negrita fiabilidad interjueces. Subrayados los valores de la matriz multirrasgo-multimétodo.

Tabla 6. Correlación entre los factores 1 y 2, y la puntuación total del cuestionario de socialización parental TXP y el Cuestionario de Análisis Clínico (CAQ) de Krug.

\begin{tabular}{|c|c|c|c|c|c|c|}
\hline \multirow[t]{2}{*}{ Dimensiones CAQ } & \multicolumn{3}{|c|}{ ADOLESCENTES } & \multicolumn{3}{|c|}{ CUIDADORES } \\
\hline & F1 & $\mathbf{F} 2$ & TOTAL & $\mathbf{F 1}$ & $\mathbf{F} 2$ & TOTAL \\
\hline Hipocondriasis &,$- 396^{\star \star}$ &,$- 209^{\star \star}$ &,$- 395^{\star \star}$ &,- 078 &,$- 133^{* *}$ &,$- 127^{\star \star *}$ \\
\hline Depresión suicida &,$- 407^{\star \star}$ &,$- 248^{\star \star}$ &,$- 417^{\star \star}$ &,$- 107^{\star}$ &,$- 155^{\star \star}$ &,$- 156^{\star \star}$ \\
\hline Agitación &,$- 131 * \star$ &,- 061 &,$- 128^{\star \star}$ &,- 050 &,$- 127^{\star \star}$ &,$- 128^{\star *}$ \\
\hline Depresión ansiosa &,$- 168^{\star \star}$ &,$- 145^{\star \star}$ &,$- 186^{\star \star}$ &,- 059 &, 036 &, 027 \\
\hline Depresión baja energía &,$- 371 * \star$ &,$- 175^{\star \star}$ &,$- 363^{\star \star}$ &,$- 091^{\star}$ &,$- 124^{\star \star}$ &,$- 123^{\star}$ \\
\hline Culpa-Resentimiento &,$- 272^{\star \star}$ &,$- 184^{\star \star}$ &,$- 285^{\star \star}$ &,- 011 &,- 046 &,- 036 \\
\hline Apatía-Retirada &,$- 307^{* *}$ &,$- 261^{\star \star}$ &,$- 339 \star \star$ &,$- 117^{\star}$ &,$- 150 * \star$ &,$- 154^{\star \star}$ \\
\hline Paranoia &,$- 409^{\star \star}$ &,- 201 ** &,$- 403 * \star$ &,- 043 &,$- 125^{\star *}$ &,$- 116^{\star}$ \\
\hline Desviación psicopática &,- 004 &,- 019 &,- 009 &,- 040 &,- 052 &,- 058 \\
\hline Esquizofrenia &,$- 390 \star \star$ &,$- 200 \star \star$ &,$- 387^{\star \star}$ &,- 073 &,- 091 &,- 089 \\
\hline Psicastenia &,$- 133^{\star \star}$ &,- 076 &,$- 135^{\star \star}$ &,- 056 &, 077 &, 055 \\
\hline Desajuste psicológico &,$- 382^{\star \star}$ &,$- 237^{\star \star}$ &,$- 393^{\star \star}$ &,- 070 &,- 071 &,- 070 \\
\hline
\end{tabular}

Nota. ${ }^{*} \mathrm{p}<0,05{ }^{* *} \mathrm{p}<0,01$

Tabla 7. Correlación entre los factores 1 y 2 del cuestionario de socialización parental TXP y el cuestionario de personalidad para adolescentes (HSPQ) de Cattell y Cattell.

\begin{tabular}{|c|c|c|c|c|c|c|}
\hline \multirow[t]{2}{*}{ Dimensiones HSPQ } & \multicolumn{3}{|c|}{ ADOLESCENTES } & \multicolumn{3}{|c|}{ CUIDADORES } \\
\hline & F1 & $\mathbf{F} 2$ & TOTAL & $\mathbf{F 1}$ & $\mathbf{F} 2$ & TOTAL \\
\hline Ansiedad &,$- 430 * \star$ &,$- 216^{\star \star}$ &,$- 427^{\star \star}$ &,- 009 &,$- 147^{\star \star}$ &,$- 134^{\star \star}$ \\
\hline Extraversión &, $087^{\star}$ &, $084^{\star}$ &, $100^{\star}$ &, 029 &,- 039 &,- 037 \\
\hline Excitabilidad &,$- 103^{\star}$ &,- 019 &,$- 092^{\star}$ &, $092^{\star}$ &, 008 &, 022 \\
\hline Independencia &,$- 166^{\star \star}$ &,- 058 &,$- 156^{\star \star}$ &,- 080 &,$- 135^{\star \star}$ &,$- 133^{\star \star}$ \\
\hline Reservado-Abierto &, $099^{\star}$ &, $102^{\star}$ &, $115^{\star \star}$ &, 038 &, 046 &, 045 \\
\hline Inteligencia &, 023 &, 041 &, 032 &,$- 097^{\star}$ &, 041 & ,015 \\
\hline Estabilidad &, $345^{\star \star}$ &, $180^{\star \star}$ &, $344^{\star *}$ &, 021 &, $096^{\star}$ &, 092 \\
\hline Calma-Excitación &,$- 240^{\star \star}$ &,- 074 &,$- 222^{\star \star}$ &,- 016 &,- 088 &,- 082 \\
\hline Sumisión-Dominancia &,$- 087^{\star}$ &,- 055 &,$- 090^{\star}$ &,$- 129^{\star}$ &,$- 112^{\star}$ &,$- 122^{\star}$ \\
\hline Entusiasmo & 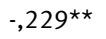 &,$- 098^{\star}$ &,- 221 ** & ,053 &,$- 163^{\star \star}$ &,$- 140 * \star$ \\
\hline Emprendedor &, $123^{\star \star}$ & ,078 &, $128^{\star *}$ &,- 031 &,- 014 &,- 022 \\
\hline Sensibilidad &, $186^{\star \star}$ &, $122^{\star \star}$ &, $194^{\star \star}$ &, 080 &, $161^{\star \star}$ &, $161^{\star \star}$ \\
\hline Seguridad &,$- 126^{\star \star}$ &,- 078 &,$- 130^{\star *}$ &, 018 &, 022 &, 026 \\
\hline Serenidad &,$- 222^{\star \star}$ &,$- 121^{\star *}$ &,$- 223^{\star *}$ &, 004 &, 006 & ,011 \\
\hline Sociabilidad &,- 030 &,- 053 &,- 043 &,- 028 & ,021 & ,019 \\
\hline Integración &, $282^{\star \star}$ &, $145^{\star \star}$ &, $281^{\star \star}$ &, 069 &, $131^{\star \star}$ &, $127^{\star \star}$ \\
\hline Relajación &,$- 229 * \star$ &,$- 137^{\star \star}$ &,$- 234^{\star *}$ &, 030 &,- 030 &,- 024 \\
\hline
\end{tabular}

Nota. ${ }^{*} \mathrm{p}<0,05{ }^{* \star} \mathrm{p}<0,01$
El $T X P-C$ presenta GLB $=0,97$ y un alfa de Cronbach de 0,87 , el factor 1 de 0,58 y el factor 2 de 0,87 .

La fiabilidad interjueces (correlaciones entre las punobservar en la tabla 5 .

La fiabilidad test-retest (coeficiente de correlación intraclase) del TXP-A fue $0,94(\mathrm{p}<0,001)$ y la del TXP-C 0,94 $(\mathrm{p}<0,001)$. tuaciones de los adolescentes y sus cuidadores) se puede 


\section{Evidencias de validez convergente y discriminante}

Respecto a las variables psicopatológicas, en las tablas 6 y 7 se pueden ver las múltiples correlaciones significativas encontradas entre las puntuaciones totales y de los dos factores, tanto del TXP-A como del TXP-C, con las puntuaciones del $C A Q$ y del $H S P Q$.

Respecto al consumo de sustancias, la correlación del POSIT con el TXP-A fue $\mathrm{r}=0,275(\mathrm{p}<0,001)$ con el factor $1, \mathrm{r}=0,140(\mathrm{p}<0,001)$ con el factor 2 y r=0,273 $(\mathrm{p}<0,001)$ con la puntuación total. Existen diferencias en el factor 1 $(\mathrm{t}=5,104 ; \mathrm{p}<0,001 ; \mathrm{TE}=0,70)$, el $2(\mathrm{t}=2,541 ; \mathrm{p}=0,011 ; \mathrm{TE}=$ $0,37)$ y la puntuación total $(\mathrm{t}=5,046 ; \mathrm{p}<0,001 ; \mathrm{TE}=0,70)$ entre los sujetos que presentan problemas con las drogas o el alcohol ( Factor $1=72,21$; DT $=14,03$; Factor $2=33,54$; $\mathrm{DT}=5,29$; Total $=105,76 ; \mathrm{DT}=16,99)$ y los que no $($ Factor $1=81,19$; DT $=11,25$; Factor $2=35,40 ; \mathrm{DT}=4,73$; Total $=$ $116,59 ; \mathrm{DT}=13,75)$.

La correlación del POSIT con el TXP-C fue $\mathrm{r}=-0,010(\mathrm{p}=$ $0,830)$ con el factor $1, \mathrm{r}=-0,127(\mathrm{p}=0,008)$ con el factor 2 y $\mathrm{r}=0,116(\mathrm{p}=0,015)$ con la puntuación total. No obstante, no existen diferencias significativas entre aquellos que presentan problemas con el alcohol y las drogas y los que no.

Respecto a los problemas de conducta, en el TXP-A, existen diferencias en las puntuaciones en el factor $1(\mathrm{t}=$ 4,084; $\mathrm{p}<0,001 ; \mathrm{TE}=2,27$ y total $(\mathrm{t}=3,259 ; \mathrm{p}=0,001 ; \mathrm{TE}=$ $1,55)$ entre los sujetos que presentan trastorno disocial ( Factor $1=57 ; \mathrm{DT}=9,05$; Total $=92,75 ; \mathrm{DT}=15,52$ ) y los que no ( Factor $1=80,66$; DT= 11,56; Total $=115,93$; DT= 14,17). Además, existe correlación del factor $1(r=-0,180$; $\mathrm{p}<0,001)$ y la puntuación total $(\mathrm{r}=-0,141 ; \mathrm{p}<0,001)$ con el número de criterios de trastorno disocial y del factor $1(\mathrm{r}=$ $-0,149 ; \mathrm{p}<0,001)$ y la puntuación total $(\mathrm{r}=-0,119 ; \mathrm{p}=0,003)$ con la gravedad del trastorno disocial. No hay diferencias en las puntuaciones según la presencia de trastorno negativista desafiante, aunque sí correlación entre el número de criterios de trastorno negativista desafiante y los factores 1 $(\mathrm{r}=-0,237 ; \mathrm{p}<0,001), 2(\mathrm{r}=-0,151 ; \mathrm{p}<0,001)$ y puntuación total $(\mathrm{r}=-0,245 ; \mathrm{p}<0,001)$.

En el TXP-C, existen diferencias en el factor $2(\mathrm{t}=2,820$; $\mathrm{p}=0,005 ; \mathrm{TE}=1,49)$ y la puntuación total $(\mathrm{t}=2,586 ; \mathrm{p}=$ $0,010 ; \mathrm{TE}=1,44)$ entre los cuidadores de adolescentes que presentan trastorno disocial ( Factor $2=42$; DT $=7,81$; Total $=61 ; \mathrm{DT}=7,93)$ y los de aquellos que no lo presentan ( Factor 2=52,84; DT =6,62; Total $=72,02 ; \mathrm{DT}=7,36)$. Además, existe correlación del factor $2(\mathrm{r}=-0,127 ; \mathrm{p}=0,008)$ y la puntuación total $(\mathrm{r}=-0,121 ; \mathrm{p}=0,011)$ con el número de criterios de trastorno disocial y del factor $2(\mathrm{r}=-0,114 ; \mathrm{p}=$ $0,017)$ y la puntuación total $(\mathrm{r}=-0,108 ; \mathrm{p}=0,024)$ con la gravedad del trastorno disocial. No existen diferencias según la presencia de trastorno negativista desafiante.

\section{Funcionamiento diferencial del cuestionario}

En el TXP-A hay correlación significativa entre los antecedentes familiares de alcoholismo y el factor $2(r=-0,088$; $\mathrm{p}=0,028)$ y la puntuación total $(\mathrm{r}=-0,085 ; \mathrm{p}=0,034)$ y los antecedentes familiares de drogodependencias y el factor $1(\mathrm{r}=-0,133 ; \mathrm{p}=0,001)$ y la puntuación total $(\mathrm{r}=-0,127 ; \mathrm{p}=$ $0,002)$.

Existen diferencias en las puntuaciones del factor $1(\mathrm{~F}=$ $3,121 ; \mathrm{p}=0,009 ; \mathrm{TE}=0,025)$ y total $(\mathrm{F}=2,706 ; \mathrm{p}=0,020 ; \mathrm{TE}=$ 0,022) según el número de hermanos. En concreto, los hijos únicos puntúan más alto en el factor 1 que los que tienen tres hermanos y los hijos únicos y los que tienen dos hermanos presentan mayor puntuación total que los que tienen tres hermanos.

No hay diferencias en las puntuaciones en el TXP-A según el género, el curso en que se encuentran, la nacionalidad y el parentesco con el cuidador principal. Respecto al género, todos los ítems son de clase AA (poco o ningún funcionamiento diferencial) excepto el 5 y el 9 que son BB (moderado funcionamiento diferencial favoreciendo ligeramente a los varones).

En el TXP-C hay correlación significativa entre los antecedentes familiares de alcoholismo y el factor $2(r=-0,131$; $\mathrm{p}=0,006)$ y la puntuación total $(\mathrm{r}=-0,125 ; \mathrm{p}=0,009)$.

Existen diferencias en el factor $2(\mathrm{~F}=3,721 ; \mathrm{p}=0,003$; $\mathrm{TE}=0,041)$ y puntuación total $(\mathrm{F}=3,809 ; \mathrm{p}=0,002 ; \mathrm{TE}=$ 0,042) según el número de hermanos. En concreto, los hijos únicos puntúan más alto que los que tienen tres hermanos en el factor 2 y la puntuación total.

No hay diferencias en el TXP-C según el género del cuidador ni del adolescente, el curso en que se encuentra el adolescente, la nacionalidad del adolescente ni el parentesco entre ellos. Respecto al género, todos los ítems son de clase AA (poco o ningún funcionamiento diferencial).

\section{Discusión}

La versión final del cuestionario de socialización parental consta de 29 ítems en TXP-A y 16 ítems en TXP-C. Se han hecho dos versiones porque desde la perspectiva bidireccional se definen las prácticas parentales como un conjunto de actitudes y tendencias globales de comportamiento parental que determina la interacción con los hijos y tiene un efecto claro en el desarrollo infantil (Escribano, Aniorte y Orgilés, 2013). Desde esta perspectiva, hay necesidad de saber no sólo la percepción que los padres tienen sobre sus prácticas parentales, sino también la perspectiva de los hijos con respecto a sus padres. Dicha información proporciona conocimiento sobre las prácticas parentales actuales desde diferentes perspectivas y es un prerrequisito para desarrollar cualquier programa de intervención (Escribano et al., 2013).

Parece que la percepción del niño del comportamiento de sus padres puede estar más relacionada con su ajuste que el comportamiento real (Schaefer, 1965) o informado de los padres (González y Landero, 2012) y que la correlación con un observador externo es mayor con los autoin- 
formes de los adolescentes que con los autoinformes de los padres (Iglesias y Romero, 2009). Además, existe evidencia de que los hijos muestran menores sesgos de deseabilidad social que los padres (García y Gracia, 2010). Sin embargo, al confiar en las informaciones de los adolescentes del estilo parental, es difícil evaluar si los padres verdaderamente usan cada estilo en la medida que los adolescentes informan que lo hacen (Trinkner, Cohn, Rebellon y Van Gundy, 2012). Por ello, es útil incluir tanto las percepciones de los adolescentes sobre el estilo parental como informes de los padres de su propio comportamiento (Trinkner et al., 2012).

En ambas versiones del cuestionario se confirma una estructura de dos factores, tal y como proponíamos en la hipótesis 1. En el TXP-A el factor 1 se denominaría afecto y comunicación (incluyendo variables afectivas, comunicativas y bajo uso del castigo y la crítica) y el factor 2 control y estructura (incluyendo roles, disciplina, normas y límites). Esta estructura bifactorial coincide con las dos dimensiones básicas encontradas en la mayor parte de estudios: tono emocional-comunicación y control-disciplina (Sansinenea y Sansinenea, 2004). Sin embargo, en nuestro estudio el factor 2 sería más amplio que la dimensión control-disciplina, pues también englobaría aspectos referentes a otro factor frecuentemente hallado en la literatura: la estructura familiar, el grado en que los padres proveen a sus hijos de un ambiente predecible, organizado y consistente (Power, 2013). En este sentido, se suele considerar que las relaciones de afecto y comunicación abierta facilitan el establecimiento de un régimen de reglas claras y bien estructuradas (García y Gracia, 2010).

Por otro lado, el uso del castigo y la crítica no saturan con el resto de variables relacionadas con control y se incluyen en el factor afecto-comunicación, quizás reflejando la distinción hallada en otros estudios entre control autoritario (comportamiento paterno altamente directivo y a menudo crítico) y control democrático (formas de control que promueven la autonomía), presentando el primero calidez baja y el segundo alta (Power, 2013). En este sentido, se ha hallado que las familias con niveles más bajos de comunicación tienden a usar más la coerción y el castigo físico (Ramírez, 2005) y el factor afecto suele incluir la aceptación parental (García y Gracia, 2010), que en nuestro caso se reflejaría en bajo uso de la crítica.

Aunque ambas versiones presentan una estructura bifactorial, uno de los factores coincide pero la composición del otro es diferente en el TXP-C, difiriendo de lo planteado en la hipótesis 1 . Así pues, el factor 2, aunque con menos ítems, se correspondería con el factor 1 del TXP-A, denominándose también afecto y comunicación pero sin incluir el castigo ni la crítica. En cambio, el factor 1 del $T X P-C$, que se compone tan sólo de cuatro ítems, no se corresponde con el factor control y estructura, sino que se denominaría valores prosociales (educación en valores, expresión de sentimientos, fomento de relaciones familiares y sociales). Las correlaciones muestran que los factores de afecto y comunicación de ambos cuestionarios son los que más se relacionan entre sí, mientras que el factor valores prosociales del TXP-C se relaciona más con el factor control y estructura del $T X P-A$. Es muy llamativo que en el $T X P-C$ no estén incluidos ítems que reflejen prácticas de disciplina y establecimiento de normas y límites, siendo lo más cercano a estas dimensiones la educación en valores prosociales. Esto podría reflejar la tendencia que parece existir en nuestro país hacia el uso de un estilo educativo más permisivo o indulgente (Fuentes et al., 2015) o que los cuidadores muestran más deseabilidad social cuando hablan de control y estructura que cuando lo hacen de afecto y comunicación, siendo por tanto estos ítems menos fiables y coherentes y más sometidos a deseabilidad social cuando los responden los cuidadores que cuando lo hacen los adolescentes (Oudhof, Rodríguez y Robles, 2012).

Además de diferir uno de los factores en ambos cuestionarios, el TXP-A incluye bastantes más ítems que el $T X P$-C. En lugar de eliminar los ítems que no están en ambas versiones o los dos factores que no coinciden para que las dos versiones sean idénticas y por tanto aumentar artificiosamente la fiabilidad interjueces, hemos optado por mantenerlos, ya que consideramos que pueden reflejar diferencias reales en la percepción de la socialización parental entre padres e hijos, ya que se ha encontrado que el nivel de acuerdo entre informantes que desempeñan el mismo rol es mayor que entre aquellos que desempeñan diferentes roles (Molinuevo, Pardo y Torrubia, 2011) y que padres e hijos tienen diferentes perspectivas de sus relaciones y conductas (Rebholz et al., 2014). De hecho, hay autores que consideran que estas discrepancias proporcionan información importante sobre las relaciones padres-hijos y pueden afectar directamente al ajuste del adolescente (Reidler y Swenson, 2012). Otros autores en cambio optan por forzar el paralelismo entre ambas versiones para permitir las comparaciones entre informantes, aunque advirtiendo de que esto puede suponer una pérdida de potencia exploratoria (Molinuevo et al., 2011). Estudios posteriores podrán valorar si mantener estos ítems y factores diferentes aporta información relevante para el desarrollo de TC y TRS.

El análisis de ítems muestra que todas las medias excepto una son mayores de 3 , lo que indica que son ítems "fáciles", la mayoría de la muestra puntúa alto en ellos. Esto es lógico dado que hablamos de población general donde la frecuencia de psicopatología es baja. Consideramos que esto permitirá una mejor discriminación entre sujetos sin patología y aquellos que presenten TRS y TC cuando se aplique el cuestionario a muestras clínicas. La mayoría de los índices de discriminación son mayores de 0,25. Hemos optado por mantener los seis ítems del TXP-A que presentan menor índice de discriminación porque también 
presentan medias más bajas que la mayoría de ítems (indicando "peor" socialización), por lo que consideramos que quizás no discriminen bien en población general pero sí puedan hacerlo en población clínica. Las medias de los residuos cuadráticos ponderadas y no ponderadas son todas menores de 2 y mayores de 0,5. En ambas versiones la mayoría de los ítems obtienen los valores recomendados: mayores de 0,5 y menores de 1,2 (Wright, Linacre, Gustafson y Martin-Löf, 1994).

Respecto a las características psicométricas del cuestionario, en el TXP-A los índices de ajuste fueron satisfactorios, a excepción del ajuste mínimo. No obstante, consideramos que el ajuste se puede considerar bueno ya que este índice es muy sensible a la presencia de curtosis y además contrasta la hipótesis nula de que los datos se ajustan "perfectamente" al modelo, lo cual es bastante improbable y hace que esta prueba sea muy restrictiva (Diamantopoulos y Siguaw, 2000). En el TXP-C, aunque el RMSR y el GFI muestran buen ajuste y el ajuste mínimo se puede justificar por lo antes expuesto, el RMSEA no es satisfactorio, por lo que el ajuste a los datos parece moderado. Los porcentajes de varianza total explicada en ambas versiones fueron similares a los encontrados para el $A C P Q$ en población española (Escribano et al., 2013). En ambas versiones el cuestionario presenta alta fiabilidad interna y excelente fiabilidad test-retest. La fiabilidad interna más baja es la presentada por el factor valores prosociales del TXP-C, aunque es similar a la hallada en algunos factores del $A C P Q$ (Escribano et al., 2013). Esta menor fiabilidad interna puede deberse al bajo número de ítems que saturan en el factor de valores prosociales o, como hemos dicho anteriormente, a que la información sobre la percepción de los hijos de los hábitos de crianza de sus padres es más fiable, coherente y menos sometida a deseabilidad social que la suministrada por sus padres (Oudhof et al., 2012).

La fiabilidad interjueces es baja, aunque similar a la encontrada en otros estudios (Escribano et al., 2013). No obstante, consideramos que esta baja concordancia entre la evaluación de los adolescentes y sus cuidadores no se debe a la calidad del cuestionario, sino a las diferencias reales entre los padres y los hijos a la hora de informar de la socialización parental encontradas también en otros estudios (Bersabé et al., 2001; González y Landero, 2012).

Respecto a las evidencias de validez, la matriz multimétodo-multirrasgo muestra adecuada validez convergente y discriminante, ya que la correlación más alta es la encontrada entre el factor 1 del TXP-A y el 2 del TXP-C (ambos evaluando afecto y comunicación). La puntuación en el factor afecto-comunicación y la total del TXP-A correlacionan significativamente con todas las variables del $C A Q$ excepto desviación psicopática. El factor control-estructura correlaciona significativamente con todas las variables excepto agitación, desviación psicopática y psicastenia. La falta de correlación con desviación psicopática podría deberse a que esta dimensión está influida especialmente por aspectos genéticos o endofenotípicos (Pardini, Raine, Erickson y Loeber, 2014) y menos por el estilo de socialización parental, a que el aprendizaje mediante el castigo está alterado en adolescentes con desviación psicopática (Salamone y Correa, 2012) o a que esta escala del CAQ parece no estar bien definida (Gómez, De Paz, Tejerina, Pérez y Luna, 2007). Tal como planteábamos en la hipótesis 2, todas las correlaciones van en la dirección esperada: a mayor puntuación en el cuestionario, menor psicopatología.

Por su parte, en el TXP-C, todas las correlaciones significativas van también en la dirección esperada en la hipótesis 2 y ambos factores y la puntuación total correlacionan significativamente con las variables de psicopatología, aunque con menos que el TXP-A. Además, el factor valores prosociales del TXP-C tan sólo correlaciona significativamente con tres de las variables (depresión suicida, depresión baja energía y apatía-retirada) lo que parece indicar que esta variable no tendría tanta relación con la psicopatología en general aunque sí con los trastornos internalizantes y los síntomas depresivos en particular, en la línea de estudios que encuentran que la conducta prosocial es un factor protector para la depresión (Llorca, Mesurado y Samper, 2014).

Como en otros estudios, las variables de socialización parental se relacionan significativamente con las variables de personalidad (Castañeda, Garrido-Fernández y Lanzarote, 2012) evaluadas con el $H S P Q$, probablemente reflejando las complejas relaciones recíprocas entre la personalidad del adolescente, su conducta, la socialización parental recibida y la socialización parental percibida (Iglesias y Romero, 2009). Aunque, otra vez, el TXP-C presenta menos relaciones significativas, especialmente el factor valores prosociales.

Respecto a las evidencias de validez de criterio, en el TXP-A los factores 1 y 2 y la puntuación total se relacionan con el consumo de drogas y alcohol y el cuestionario diferencia entre aquellos que presentan problemas con las drogas y el alcohol y los que no. Además, el factor afecto-comunicación y la puntuación total se relacionan con el trastorno disocial y diferencian entre los que lo presentan y los que no. Ambos factores y la puntuación total se relacionan con la presencia de síntomas de trastorno negativista desafiante, aunque el cuestionario no llega a diferenciar los que presentan el trastorno de los que no. Esto coincide con los estudios que encuentran que la calidez y el control conductual se asocian con niveles más bajos de problemas de conducta en la adolescencia (Trudeau et al., 2012), que menos calidez emocional y más rechazo parental se asocian con el abuso de sustancias y que la cohesión y la adaptabilidad se relacionan negativamente con el alcoholismo (Abasi y Mohammadkhani, 2016). Otros autores también encuentran que el control parental es un factor protector contra el abuso de alcohol y otros problemas en la adolescencia (Cabanillas-Rojas, 2012). 
En el $T X P-C$, el factor afecto y comunicación y la puntuación total se relacionan con el consumo de drogas y alcohol, aunque el cuestionario no llega a diferenciar los que presentan problemas con las drogas y el alcohol y los que no. El factor afecto y comunicación y la puntuación total se relacionan con el trastorno disocial y el cuestionario diferencia entre los adolescentes que presentan trastorno disocial y los que no. Sin embargo, no existen relaciones entre el cuestionario y el trastorno negativista desafiante.

Todos estos datos apoyan que el TXP-A mide aquello para lo que fue diseñado: prácticas de socialización parental relacionadas con la presencia de TRS y TC. Sin embargo, en el caso del TXP-C, si bien el factor afecto-comunicación sí parece tener evidencias de validez, el factor valores prosociales no se relaciona con los TRS ni los TC. Esto puede deberse a que la percepción del adolescente de las prácticas parentales educativas (relacionadas con control-estructura en el $T X P-A$ y con valores prosociales en el TXP-C) se relaciona más que la del cuidador con la psicopatología en general, los TC y los TRS (González y Landero, 2012); pero también podría ser que, al ser el adolescente el que cumplimenta el CAQ y el POSIT y responde a la entrevista sobre los TC, la correlación de estos criterios externos con el TXP-A es mayor al ser el mismo informante el que los cumplimenta (Molinuevo et al., 2011). En estudios futuros se podría considerar evaluar los TC y TRS desde la perspectiva de los cuidadores para comprobar si la correlación aumenta o se mantiene el resultado de que la percepción de los cuidadores de su socialización en valores prosociales no se relaciona con la presencia de TRS y TC.

Destacar que no se hallaron diferencias en ninguna de las dos versiones entre aquellos que presentaban trastorno negativista desafiante y los que no. Esto puede deberse a la escasez de sujetos con este diagnóstico en la muestra o a que el cuestionario está diseñado pensando en trastornos de conducta graves, presentado el trastorno negativista desafiante menos gravedad que el trastorno disocial (American Psychiatric Association, 2014), del que también hay pocos sujetos en la muestra pero aún así se hallan diferencias. Dado que la validación se ha realizado con población general, hay muy pocos sujetos en la muestra con TC y relativamente pocos con uso problemático de drogas y alcohol. Además, podría ser que los adolescentes que no han participado en el estudio sean precisamente los que presenten más psicopatología y/o experimenten prácticas parentales más disfuncionales. Sería interesante realizar un estudio de validación en población clínica para así poder estudiar mejor qué prácticas parentales se relacionan más con los problemas de conducta y el uso de drogas. Esto permitiría realizar estudios longitudinales que permitieran hallar puntos de corte para detectar a aquellos sujetos con alta probabilidad de presentar TC y TRS, lo que permitiría realizar intervenciones preventivas.
Destacar por último que ambas versiones del cuestionario no presentan funcionamiento diferencial que limite su aplicación, pues no se encuentran sesgos según género, curso, nacionalidad y parentesco con el cuidador. El resto de diferencias de funcionamiento encontradas creemos que no constituyen una limitación sino que reflejan la relación lógica ya descrita en la literatura entre las variables de socialización familiar y el resto de variables. Así, a mayor edad del adolescente, menor estructuración, disminuyen las normas y límites para fomentar la autonomía (Oliva, 2006); la presencia de antecedentes familiares de alcoholismo y drogodependencias se relaciona con peor socialización parental (Slesnick, Feng, Brakenhoff y Brigham, 2014) y un mayor número de hijos se relaciona con una peor percepción del clima emocional, quizás por el menor tiempo que puede dedicar el cuidador a cada hijo (Beltrán, 2013).

\section{Limitaciones}

La principal limitación del estudio es que no se ha usado otro cuestionario de socialización parental para evaluar la validez convergente. Esto se ha hecho, además de por las limitaciones que presentan dichas escalas (Bersabé et al., 2001), porque la mayoría de escalas están diseñadas para evaluar estilos de socialización parental en general, mientras que a nosotros nos interesaba un instrumento que evaluara prácticas parentales concretas que se relacionaran con la presencia de TRS y TC. Siguiendo a Mâsse y Watts (2013), el estilo parental general refleja las actitudes y creencias parentales que crean el clima emocional global en el cual tienen lugar las interacciones padres-hijos; el estilo parental es descrito mediante tipologías que clasifican a los padres basándose en sus niveles de capacidad de respuesta y exigencia (autoritario, democrático, permisivo y negligente); mientras que las prácticas parentales son las estrategias específicas que los padres usan para alcanzar resultados deseados. Aunque hay autores que consideran que los estilos parentales presentan una correspondencia más clara con los ajustes psicosociales de los hijos que las prácticas parentales (García y Gracia, 2010), nos parece que evaluar las prácticas parentales concretas que se relacionan con la presencia de los trastornos es mucho más útil para diseñar estrategias de prevención e intervención. Dado que la definición de los factores parentales en la literatura carece de consistencia, frecuentemente se solapan conceptualmente, limitando la comprensión de qué estrategias parentales específicas son efectivas en reducir el consumo de sustancias en adolescentes (Ryan, Jorm y Lubman, 2010). Por ello, es necesario para la investigación establecer variables parentales definidas y distintas con métodos consistentes de evaluación (Ryan et al., 2010).

Una posible limitación es la tasa de abandonos, pues podría ser que los que no aceptan participar en el estudio sean precisamente los adolescentes que presentan más psi- 
copatología y los cuidadores que abandonan los que presentan prácticas de socialización parental menos efectivas. No obstante, el $23 \%$ de abandonos encontrados en cuidadores es bastante inferior al 36\% del estudio de Bersabé et al. (2001) y al 49,7\% del estudio de Molinuevo et al. (2011), por lo que parece un porcentaje aceptable.

Otra posible limitación es haber centrado la evaluación de la socialización parental únicamente en el cuidador principal. Se ha elegido esta opción por varias razones. En la actualidad el número de hogares monoparentales está aumentando (Instituto Nacional de Estadística, 2016), por lo que en algunos casos no procede preguntar por ambos progenitores. Además, aunque la mayor parte de la literatura suele focalizar en la socialización de la madre, también los padres juegan un rol significativo en el desarrollo de los hijos y, en muchos hogares y culturas, otros familiares, amigos y proveedores de cuidados también contribuyen a dicho desarrollo (Lomanowska, Boivin, Hertzman y Fleming, 2017). Creemos que centrándonos en la persona a quien el adolescente considera su cuidador principal se optimiza la información, sobre todo porque la correspondencia encontrada entre los estilos educativos de ambos progenitores ha sido baja (Winsler, Madigan y Aquilino, 2005).

Otra limitación es que, si bien se ha considerado tanto la perspectiva del adolescente como la del cuidador, no se han considerado las complejas relaciones interactivas entre las conductas de ambos y la aparición y mantenimiento de TC y TRS. La socialización parental es un proceso transaccional bidireccional, donde el comportamiento y la psicopatología del adolescente cambia también el comportamiento parental (Kerr, Stattin y Özdemir, 2012). Futuros estudios longitudinales podrían modelar estas relaciones mediante ecuaciones estructurales.

Son también limitaciones del estudio las relacionadas con el uso de cuestionarios de autoinforme: sesgo de deseabilidad social, sesgos de recuerdo, limitada conciencia del comportamiento de uno mismo y respuestas descuidadas o aleatorias (Power et al., 2013).

\section{Conclusiones}

El $T X P$ - $C$ parece un instrumento fiable y sin sesgos, pero no ha aportado de momento pruebas suficientes de validez. El factor afecto-comunicación parece relacionarse con psicopatología en general, TC y TRS; mientras que el factor valores prosociales sólo se relaciona con depresión pero no lo hace con TRS y TC. Estudios posteriores usando instrumentos de evaluación de TC y TRS informados por el cuidador o terceros permitirían confirmar estas relaciones del factor afecto-comunicación y si la percepción del cuidador del factor valores prosociales se relaciona o no con TRS y TC en el adolescente.

El TXP-A parece un instrumento fiable, válido y sin sesgos para evaluar la percepción de las prácticas de socializa- ción parental relacionadas con la aparición de TC y TRS en adolescentes de entre 14 y 16 años. Está validado en población general y parece pertinente validarlo en otros grupos de edad y en población clínica. La realización de estudios longitudinales con este instrumento podría permitir establecer puntos de corte para detectar población en riesgo de desarrollar TC y TRS, identificar las prácticas parentales más relacionadas con los TC y TRS y focalizar en ellas las intervenciones preventivas.

\section{Reconocimientos}

Al Profesor Miguel Ángel Catalá de la Universidad de Valencia por regalarnos la idea inicial de este estudio.

Estudio financiado principalmente por la Consellería de Sanidad, Generalitat Valenciana, referencias SMI 09/2009 y SMI 28/2011, así como por la Universidad CEU-Cardenal Herrera. La financiación para el Open Access fue aportada por la Fundación de Investigación C.V. del Hospital Provincial de Castellón (Referencia: CAF-17-065).

\section{Conflicto de intereses}

No existen conflictos de intereses. El cuestionario no tiene copyright y su uso será gratuito.

\section{Referencias}

Abad, F. J., Olea, J., Ponsoda, V. y García, C. (2011). Medición en ciencias sociales y de la salud. Madrid: Síntesis.

Abasi, I. y Mohammadkhani, P. (2016). Family Risk Factors Among Women With Addiction-Related Problems: An Integrative Review. International Journal of High Risk Behaviors and Addiction, 5, e27071. doi:10.5812/ijhrba.27071.

American Psychiatric Association (2002). DSM-IV-TR. Manual diagnóstico y estadístico de los trastornos mentales. Texto revisado. Barcelona: Masson.

American Psychiatric Association (2014). Manual diagnóstico y estadístico de los trastornos mentales, $5^{a}$ edición (DSM-5). Arlington, VA: American Psychiatric Association.

Araujo, M., Golpe, S., Braña, T., Varela, J. y Rial, A. (2018). Validación psicométrica del POSIT para el cribado del consumo de riesgo de alcohol y otras drogas entre adolescentes, Adicciones. 30, 130-139. doi:10.20882/adicciones.0.0.

Arrindel, W. A., Akkerman, A., Bagés, N., Feldman, L., Caballo, V., Oei, T. P. S.,..., Zaldívar, F. (2005). The short EMBU in Australia, Spain and Venezuela: factor invariance and associattions with sex roles, self-esteem and Eysenckian personality dimensions. European Journal of Psychological Assessment, 21, 56-66.

Baumrind, D. (1991). The influence of parenting style on adolescent competence and substance use. Journal of Early Adolescence, 11, 56-95. 
Beltrán, A. (2013). El tiempo de la familia es un recurso escaso:¿cómo afecta su distribución en el desempeño escolar? Apuntes Centro de Investigación de la Universidad del Pacífico, 72, 117-156.

Bersabé, R., Fuentes, M. J. y Motrico, E. (2001). Análisis psicométrico de dos escalas para evaluar estilos educativos parentales. Psicothema, 13, 678-684.

Bravo, M. L. y Arrieta, J. J. (2005). El método Delphi. Su implementación en una estrategia didáctica para la enseñanza de las demostraciones geométricas. Revista Iberoamericana de Educación, 35.

Buri, J. R. (1991). Parental Authority Questionnaire. Journal of Personality Assessment, 57, 110-119.

Cabanillas-Rojas, W. (2012). Factores de riesgo/protección y los programas preventivos de drogodependencias en el Perú. Revista Peruana de Medicina Experimental y Salud Pública, 29, 104-111. doi:10.17843/rpmesp.2012.291.316.

Castañeda, A., Garrido-Fernández, M. y Lanzarote, M. D. (2012). Menores con conducta de maltrato hacia los progenitores: un estudio de personalidad y estilos de socialización. Revista de Psicología Social, 27, 157-167. doi:10.1174/021347412800337933.

Cattell, M. D. y Cattell R. B. (1981). HSPQ Cuestionario de Personalidad para Adolescentes. Madrid: TEA Ediciones.

Cattell, R. B. y Cattell, H. E. P. (1995). Personality structure and the new fifth edition of the 16PF. Educational and Psychological Measurement, 6, 926-937.

Diamantopoulos, A. y Siguaw, J. A. (2000). Introducing Lisrel: A Guide for the Uninitiated. London: SAGE Publications.

Escribano, S., Aniorte, J. y Orgilés, M. (2013). Factor structure and psychometric properties of the Spanish version of the Alabama Parenting Questionnaire (APQ) for children. Psicothema, 25, 324-329. doi:10.7334/psicothema2012.315.

Fernández, M. P. (2009). Los estilos educativos de padres y madres. Innovación y experiencias educativas, 16, 1-9.

Forns, M., Amador, J. A., Abad, J. y Martorell, B. (1998). El cuestionario de análisis clínico (CAQ): fiabilidad y valor discriminante en una muestra clínica. Revista de Psicopatología y Psicología Clínica, 3, 27-38.

Fuentes, M. C., García, F., García, E. y Alarcón, A. (2015). Los estilos parentales de socialización y el ajuste psicológico. Un estudio con adolescentes españoles. Revista Psicodidáctica, 20, 117-138. doi:10.1387/RevPsicodidact.10876.

García, F. y Gracia, E. (2010). ¿Qué estilo de socialización parental es el idóneo en España? Un estudio con niños y adolescentes de 10 a 14 años. Infancia y aprendizaje, 33, 365-384.

Gómez, J., De Paz, R., Tejerina, M. C., Pérez, M. D. y Luna, A. (2007). Aplicación de los tests 16 PF y CAQ a una escala de valoración del daño psicológico. Revista Portuguesa do Dano Corporal, 17, 51-78. doi:10.14195/16478630_17_4.
González, M. y Landero, R. (2012). Diferencias en la percepción de estilos parentales entre jóvenes y adultos de las mismas familias. Summa Psicológica UST, 9, 53-64.

Hair, F., Anderson, R. E, Tatham, R. L. y Black, W. C. (1998). Multivariate data analysis with readings. New Jersey: Prentice Hall, 5th edition.

Holmes, S., Slaughter, J. y Kashani, J. (2001). Risk factors in childhood that lead to the development of conduct disorder and antisocial personality. Child Psychiatry and Human Development, 31, 183-193.

IBM Corp. Released. (2011). IBM SPSS Statistics for Windows, Version 20.0. Armonk, NY: IBM Corp.

Iglesias, B. y Romero, E. (2009). Estilos parentales percibidos, psicopatología y personalidad en la adolescencia. Revista de Psicopatología y Psicología Clínica, 14, 63-77.

Instituto Nacional de Estadística (2016). Encuesta continua de hogares. Año 2015. Recuperado de http:/ / www.ine.es/ prensa/np965.pdf.

Kerr, M., Stattin, H. y Özdemir, M. (2012). Perceived Parenting Style and Adolescent Adjustment: Revisiting Directions of Effects and the Role of Parental Knowledge. Developmental Psychology, 48, 1540-1553.

Krug, S. (1994). CAQ. Cuestionario de análisis clínico. Madrid: TEA ediciones.

Lidner, M. (2013). Associations between parenting style, physical discipline, and adjustment in adolescents' reports. Psychological Reports: Disability and Trauma, 112, 933-975. doi:10.2466/15.10.49.PR0.112.3.933-975.

Llorca, A., Mesurado, B. y Samper, P. (2014). El rol mediador de la empatía, la conducta prosocial y la conducta agresiva en la depresión y la ansiedad. Ansiedad y Estrés, 20, 245-256.

Loke, A. Y. y Mak, Y. (2013). Family process and peer influences on substance use by adolescents. International Journal of Environmental Research and Public Health, 10, 3868-3885. doi:10.3390/ijerph10093868.

Lomanowska, A. M., Boivin, M., Hertzman, C. y Fleming, A. S. (2017). Parenting begets parenting: A neurobiological perspective on early adversity and the transmission of parenting styles across generations. Neuroscience, 342, 120-139. doi:10.1016/j.neuroscience.2015.09.029.

Lorenzo-Seva, U. y Ferrando, P. J. (2006). FACTOR: A computer program to fit the exploratory factor analysis model. Behavioral Research Methods, Instruments and Computers, 38, 88-91.

Maccoby, E. y Martin, J. (1983). Socialization in the context of the family: parent-child interaction. En: E.M., Hetherington (Ed.), Handbook of child psychology: socialization, personality, and social development (pp. 1-102). Nueva York: Wiley.

Martínez, I., Fuentes, M. C., García, F. y Madrid, I. (2013). El estilo de socialización familiar como factor de prevención o de riesgo para el consumo de sustancias y otros problemas de conducta en adolescentes españoles. Adicciones, 25, 235-242. doi:10.20882/adicciones.51. 
Martínez, J. F., Díaz, A., Salazar, A. A. y Duron, M. (2014). Study of parental models: building an instrument for their exploration. Revista da Escola de Enfermagem - USP, 48, 594-601. doi:10.1590/S0080-623420140000400004.

Mâsse, L. C. y Watts, A. W. (2013). Stimulating innovations in the measurement of parenting constructs. Childhood Obesity, 9, 5-13. doi:10.1089/chi.2013.0041.

Meyer, J. P. (2014). Applied measurement with jMetrick. New York: Routledge Taylor \& Francis.

Mezquita, L., Moya, J., Edo, S., Maestre, E., Viñas, M., Ortet, G. y Ibáñez, M. I. (2006). Personalidad y estilos educativos parentales como predictores del consumo de alcohol en adolescentes. Forum de Reserca, 12. Recuperado de http://hdl.handle.net/10234/78550.

Molinuevo, B., Pardo, Y. y Torrubia, R. (2011). Psychometric Analysis of the Catalan Version of the Alabama Parenting Questionnaire (APQ) in a Community Sample. The Spanish Journal of Psychology, 14, 944-955.

Moos, R. H., Moos, B. S. y Trickett, E. J. (1984). Escalas de Clima Social. Madrid: TEA.

Musitu, G., y García, J. F. (2001). ESPA29. Escala de socialización parental en la adolescencia. Madrid: TEA Ediciones.

National Institute on Drug Abuse (1991). The adolescent assessment/referral system. Rockville: NIDA.

Oliva, A. (2006). Relaciones familiares y desarrollo adolescente. Anuario de Psicología, 37, 209-223.

Oliva, A., Parra, A., Sánchez-Quija, I. y López, F. (2007). Estilos educativos materno y paterno: evaluación y relación con el ajuste del adolescente. Anales de Psicología, 23, 49-56.

Olson, D. H., Portner, J. y Lavee, Y. (1985). Manual de la Escala de Cohesión y Adaptabilidad Familiar (FACES III Manual). Minneapolis: Life Innovation.

Olson, D. H., Rusell C. S. y Sprenke, D. H. (1988). Circumplex model: systematic assessment and treatment of families. New York: The Haworth Press.

Oudhof, H., Rodríguez, B. y Robles, E. (2012). La percepción de la crianza en padres, madres e hijos adolescentes pertenecientes al mismo núcleo familiar. Liberabit, $18,75-82$.

Pardini, D. A., Raine, A, Erickson, K. y Loeber, R. (2014). Lower Amygdala Volume in Men is Associated with Childhood Aggression, Early Psychopathic Traits and Future Violence. Biological Psychiatry, 75, 73-80. doi:10.1016/j. biopsych.2013.04.003.

Pérez, P. M. (2012). La socialización parental en padres españoles con hijos de 6 a 14 años. Psicothema, 24, 371-376.

Power, T. G. (2013). Parenting Dimensions and Styles: A Brief History and Recommendations for Future Research. Childhood Obesity, 9, S14-S21. doi:10.1089/ chi.2013.0034.

Power, T. G., Sleddens, E. F. C., Berge, J., Connell, L., Govig, B., Hennessy, E.,..., St. George, S. M. (2013). Contemporary Research on Parenting: Conceptual, Metho- dological, and Translational Issues. Childhood Obesity, 9, S87-S94. doi:10.1089/chi.2013.0038.

Ramírez, M. A. (2005). Padres y desarrollo de los hijos: prácticas de crianza. Estudios Pedagógicos XXXI, 2, 167177. doi:10.4067/S0718-07052005000200011.

Rebholz, C. E., Chinapaw, M. J. M., van Stralen, M. M., Bere, E., Bringolf, B., De Bourdeaudhuij, I.,..., te Velde, S. J. (2014). Agreement between parent and child report on parental practices regarding dietary, physical activity and sedentary behaviours: the ENERGY cross-sectional survey. BMC Public Health, 14, 918. doi:10.1186/14712458-14-918.

Rees, R. y Valenzuela, A. (2003). Características individuales y de la estructura familiar de un grupo de adolescentes abusadores de alcohol y/o marihuana. Revista Chilena Neuro-Psiquiatría, 41, 173-185. doi:10.4067/ S0717-92272003000300002.

Reidler, E. B. y Swenson, L. P. (2012). Discrepancies Between Youth and Mothers' Perceptions of Their Mother-Child Relationship Quality and Self-Disclosure: Implications for Youth- and Mother-Reported Youth Adjustment. Journal of Youth and Adolescence, 41, 1151-1167. doi:10.1007/s10964-012-9773-8.

Rodríguez, A. y Torrente, G. (2003). Interacción familiar y conducta antisocial. Boletín de Psicología, 78, 7-19.

Romero, E., Rodríguez, C., Villar, P. y Gómez-Fraguela, A. (2017). Intervención sobre problemas de conducta tempranos como prevención indicada del consumo de drogas: siete años de seguimiento. Adicciones, 29, 150-162. doi:10.20882/adicciones.722.

Rosa-Alcázar, A. I., Parada-Navas, J. L. y Rosa-Alcázar, A. (2014). Síntomas psicopatológicos en adolescentes españoles: relación con los estilos parentales percibidos y la autoestima. Anales de Psicología, 30, 133-142. doi:10.6018/analesps.30.1.165371.

Ryan, S. M., Jorm, A. F. y Lubman, D. I. (2010). Parenting factors associated with reduced adolescent alcohol use: a systematic review of longitudinal studies. Australian and New Zealand Journal of Psychiatry, 44, 774-783. doi:10 $.1080 / 00048674.2010 .501759$.

Salamone, J. D. y Correa, M. (2012). The Mysterious Motivational Functions of Mesolimbic Dopamine. Neuron, 76, 470-485. doi:10.1016/j.neuron.2012.10.021.

Sansinenea, P. y Sansinenea, E. (2004). Autoestima y desaprobación parental. Psiquis, 25, 167-175.

Schaefer, E. (1965). Child's Reports of Parental Behavior: An Inventory. Child Development, 36, 413-424.

Shelton, K. K., Frick, P. J. y Wootten, J. (1996). Assessment of parenting practices in families of elementary schoolage children. Journal of Clinical Child Psychology, 25, 317329.

Slesnick, N., Feng, X., Brakenhoff, B. y Brigham, G. S. (2014). Parenting under the influence: the effects of opioids, alcohol and cocaine on mother-child interac- 
tion. Addictive Behaviors, 39, 897-900. doi:10.1016/j.addbeh.2014.02.003.

Tornay, L., Michaud, P. A., Gmel, G., Wilson, M. L., Berchtold, A. y Surís, J. C. (2013). Parental monitoring: a way to decrease substance use among Swiss adolescents? European Journal of Pediatrics, 172, 1229-1234. doi:10.1007/ s00431-013-2029-0.

Trinkner, R., Cohn, E. S., Rebellon, C. J. y Van Gundy, K. (2012). Don't trust anyone over 30: Parental legitimacy as a mediator between parenting style and changes in delinquent behavior over time. Journal of Adolescence, 35, 119-132. doi:10.1016/j.adolescence.2011.05.003.

Trudeau, L., Mason, W. A., Randall, G. K., Spoth, R. y Ralston, E. (2012). Effects of Parenting and Deviant Peers on Early to Mid-Adolescent Conduct Problems. Journal Abnormal Children Psychology, 40, 1249-1264. doi:10.1007/ s10802-012-9648-1.

Visser, L., de Winter, A. F., Vollebergh, W. A. M., Verhulst, F. C. y Reijneveld, S. A. (2013). The Impact of Parenting Styles on Adolescent Alcohol Use: The TRAILS Study. European Addiction Research, 19, 165-172. doi:10.1159/000342558.

Waller, R., Gardner, F. y Hyde, L. W. (2013). What are the associations between parenting, callous-unemotional traits, and antisocial behavior in youth? A systematic review of evidence. Clinical Psychology Review, 33, 593-608. doi:10.1016/j.cpr.2013.03.001.

Winsler, A., Madigan, A. L. y Aquilino, S. A. (2005). Correspondence between maternal and paternal parenting styles in early childhood. Early Childhood Research Quarterly, 20, 1-12. doi:10.1016/j.ecresq.2005.01.007.

Wright, B. D., Linacre, J. M., Gustafson, J. E. y Martin-Löf, P. (1994). Reasonable mean-square fit values. Rasch Measurement Transactions, 8, 370. 